\title{
PHENOLICS OF CYPERUS ALOPECUROIDES ROTTB. INFLORESCENCES AND THEIR BIOLOGICAL ACTIVITIES
}

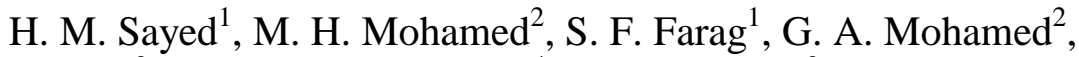
R. $\mathrm{Ebel}^{3}$, O. R. M. Omobuwajo ${ }^{4}$ and P. Proksch ${ }^{3}$

${ }^{1}$ Department of Pharmacognosy, Faculty of Pharmacy, Assiut University, Assiut, Egypt

${ }^{2}$ Department of Pharmacognosy, Faculty of Pharmacy, Al-Azhar University, Assiut, Egypt

${ }^{3}$ Institute of Pharmaceutical Biology and Biotechnology, HeinrichHeine-University, Universitätsstraße 1, 40225 Düsseldorf, Germany

${ }^{4}$ Department of Pharmacognosy, Faculty of Pharmacy, Obafemi Awolowo University, Ile-Ife, Nigeria

تم فى هذا البحث فصل ستة عشر مركبا فينولبا من خلاصة

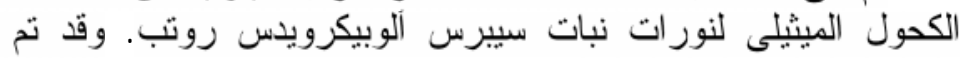

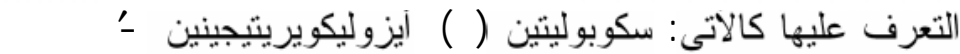

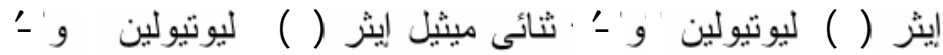

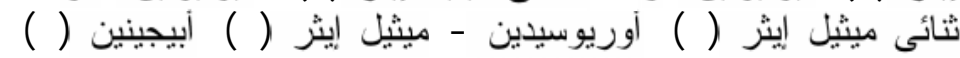

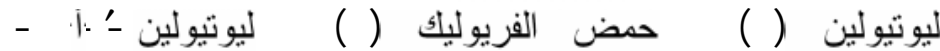

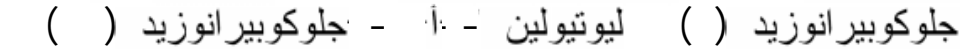

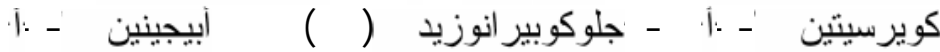

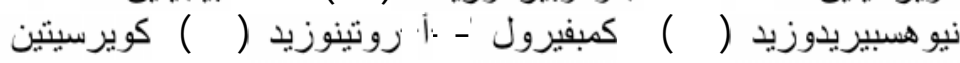

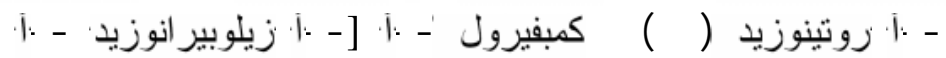

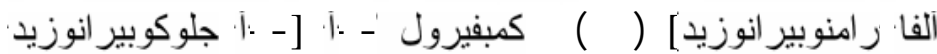

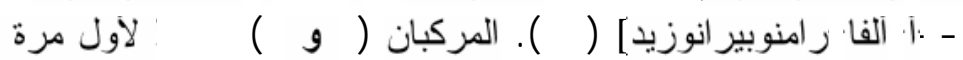

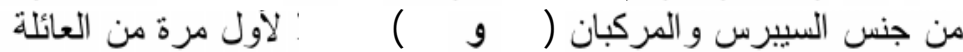

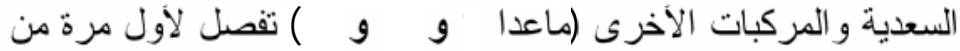

وقد تم التعرف على التركيب الدقيق لهذه المركبات بدراسة

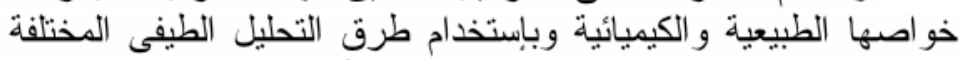

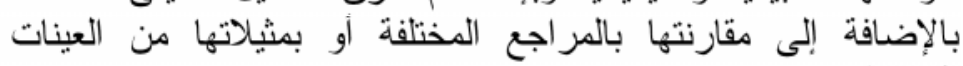

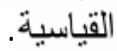

Received in 7/8/2005 \& Accepted in 22/9/2005 


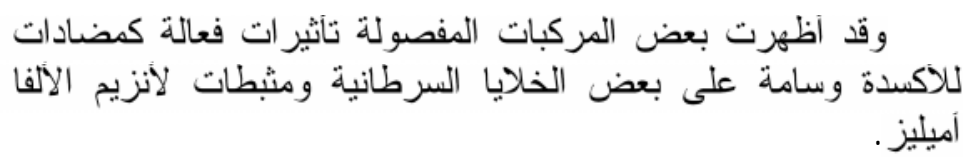

Sixteen phenolic compounds, scopoletin (1), isoliquiritigenin 4'methyl ether (2), luteolin 5,3'-dimethyl ether (3), luteolin 7,3'dimethyl ether (4), aureusidin 4-methyl ether (5), apigenin (6), luteolin (7), trans-ferulic acid (8), luteolin 4'-O- $\beta$-D-glucopyranoside (9), luteolin 7-O- $\beta$-D-glucopyranoside (10), quercetin 3-O- $\beta$-D-glucopyranoside (11), apigenin 7-O-neohesperidoside (12), kaempferol 3-O-rutinoside (13), quercetin 3-O-rutinoside (14), kaempferol 3-O-[2-O-D-xylopyranosyl-6-O- $\alpha$-L-rhamnopyranosyl]- $\beta$-D-glucopyranoside (15) and kaempferol 3-O-[2-OD-glucopyranosyl-6-O- $\alpha$-L-rhamnopyranosyl]- $\beta$-D-gluco-

pyranoside (16) were isolated from the methanolic extract of the inflorescences of Cyperus alopecuroides Rottb. for the first time. Their structures have been established on the basis of physical, chemical and spectroscopic methods in addition to comparison with literature data and/or authentic samples. The antioxidant and cytotoxic activities in addition to $\alpha$-amylase inhibitory activity of the isolated compounds have been studied.

\section{INTRODUCTION}

The sedge family, Cyperaceae comprises about 4000 species within 90 genera. Cyperus is the largest genus that includes about 550 species. ${ }^{1}$

Cyperus alopecuroides Rottb. (Foxtail sedge; Samar) is a perennial stout leafy herb, reaching up to 150 $\mathrm{cm}$ high, having triangular culm, broad flat leaves, large numerous lanceolate inflorescences and widely distributed in tropical areas. In Egypt, it is naturally growing at the Nile Delta, the borders between fields and also cultivated in limited areas for commercial manufacture of mat and traditional chair-making. ${ }^{2}$

Previous phytochemical studies on Cyperus species led to the isolation of quinones, ${ }^{3-5}$ flavonoids, ${ }^{6-9}$ coumarins, ${ }^{10 \& 11}$ furanochromones, ${ }^{12}$ sesquiterpenes, ${ }^{13-17}$ alkaloids, ${ }^{18}$ saponins, ${ }^{19}$ sterols ${ }^{19 \& 20}$ fatty acids ${ }^{20}$ and phenolic acids. $^{21}$

Many Cyperus species are used as foods. In folk medicine, they are used as spermatogenic, aphrodisiac, galactagogue, pectoral, emollient, anthelmintic, diaphoretic, astringent, digestive tonic, anti-inflammatory, antispasmodic, antirheumatic and hepatoprotective. Also, they are used 
as diuretic and to promote menstruation. ${ }^{17,22-25}$

The ethanolic extract of the overground parts of Cyperus alopecuroides Rottb. produced signs of pain and allergy on rabbit's skin. ${ }^{26 \& 27}$ These results revealed that the extract contained histamine or histamine like substance. ${ }^{26 \& 27}$ Also, the ethanolic and the ethereal extracts of the overground parts showed antimicrobial activity ${ }^{10 \& 27}$ while the ethanolic extract of the inflorescences showed moderate estrogenic activity. $^{28}$

Concerning the diversity of chemical classes of $C$. alopecuroides Rottb., several previous reports were cited: sesquiterpenes and a diterpene from the essential oil of the tubers and rhizomes; ${ }^{15,29 \& 30}$ quinones from the rhizomes and the inflorescences; ${ }^{3 \& 28}$ coumarins from the aerial parts ${ }^{10}$ in addition to flavonoids from the leaves and inflorescences $6 \& 28$ were isolated. Also, the carbohydrate, lipid, protein and amino acid contents from the overground parts of the plant were studied. ${ }^{26}$ In the present paper, we wish to describe the isolation and characterization in addition to investigation of the biological activities of the phenolic constituents of the inflorescences of this plant.

\section{EXPERIMENTAL}

\section{General procedures}

The UV spectra were carried out in methanol (Merck) using a PerkinElmer Lambda 25 UV/VIS spectrophotometer. Electron impact mass spectra (EIMS) were recorded on a Finnigan MAT TSQ 7000 mass spectrometer. Positive-ion electrospray ionization mass spectra (ESIMS) were performed on a Thermofinnigan LCQ DECA mass spectrometer coupled to an Agilent 1100 HPLC system equipped with a photodiode-array detector. Gas chromatographic (GC) analysis was carried out using an Agilent 6850 series gas chromatograph coupled to a flame ionization detector (FID) and HP-5 column (cross linked 5\% PHM/Siloxan, $30 \mathrm{~m} \times 250 \mu \mathrm{m}$ diameter $\times 0.25 \mu \mathrm{m}$ film thickness, Macherey und Nagel, Düren, Germany). ${ }^{1} \mathrm{H}$ - and ${ }^{13} \mathrm{C}-\mathrm{NMR}$ spectra were measured on Brüker DRX 500 spectrometer (Brüker, Rheinstetten, Germany). High performance liquid chromatography was performed on a HPLC system (Merck, Darmstadt, Germany) consisting of a LachromMerck Hitachi L-7100 pump and an L-7400 UV detector using a C-18 column $(250 \times 8 \mathrm{~mm}$ i.d., prefilled with Eurospher 100, Knauer, Berlin, Germany), a flow rate $5.0 \mathrm{ml} / \mathrm{min}$, UV detection at $\lambda 280 \mathrm{~nm}$ and HPLC gradient programm: 60:40 methanol/water at 0 and $5 \mathrm{~min}$; 100:0 methanol/water at 38 and $45 \mathrm{~min}$. Vacuum liquid chromatography (VLC) was carried out on silica gel 60 (0.04-0.063 mm, 500 g, Merck). Column chromatographic separations were performed over silica gel 60 (0.040-0.063 mm, Merck), Sephadex LH-20 (0.25-0.1 mm, Merck) and RP18 columns (40-63 $\mu \mathrm{m}$, Merck). TLC analyses were carried out on precoated silica gel $\mathrm{F}_{254}$ aluminium 
sheets and RP-18 $\mathrm{F}_{254} \mathrm{~s}$ glass plates (Merck). Compounds were detected by UV absorption at $\lambda 254$ and 366 $\mathrm{nm}$ followed by spraying with anisaldehyde $/ \mathrm{H}_{2} \mathrm{SO}_{4}$ reagent and heating at $110^{\circ} \mathrm{C}$ for $1-2 \mathrm{~min}$. The solvent systems used for TLC analyses were: dichloromethanemethanol (9.5:0.5, solvent system I), dichloromethane-methanol $\quad(9: 1$, solvent system II), dichloromethanemethanol (8:2, solvent system III), dichloromethane-methanol $\quad(6: 4$, solvent system IV) and $n$ butanol-acetone-formic acid-water (60:17:8:15, solvent system V). Authentic flavonoids were obtained from the Institute of Pharmaceutical Biology and Biotechnology, Heinrich -Heine-University, Universitätsstraße 1, 40225 Düsseldorf, Germany. 1,1Diphenyl-2-picrylhydrazyl radical $(\mathrm{DPPH})$, propyl gallate (PG) and reference sugars were purchased from Sigma Chemical Co. (Germany). The EnzCheck Amylase Assay Kit (E11954) was purchased from Molecular Probes (GmbH, Germany). Acarbose (Ac) was purchased from Kohlpharma (GmbH, Germany).

\section{Plant material}

The inflorescences of Cyperus alopecuroides Rottb. were collected in July 2002 from plants growing on the margins of the ponds and banks of the Nile-River, Mankabad, Assiut, Egypt. The plant was kindely identified by Prof. Dr. Salah ElNagar, Professor of Plant Taxonomy, Faculty of Science, Assiut University, Assiut, Egypt. A voucher specimen has been deposited at the Herbarium of Pharmacognosy Department, Faculty of Pharmacy, Assiut University, Assiut, Egypt.

\section{Extraction and isolation}

The air-dried powdered inflorescences of $C$. alopecuroides Rottb. $(2 \mathrm{~kg})$ were extracted with methanol $(6 \mathrm{~L} \times 4)$ at room temperature. The combined extract was concentrated under reduced pressure to afford a dark brownish green residue $(130.0 \mathrm{~g})$. The latter was suspended in distilled water (500 $\mathrm{ml})$ then partitioned with $n$-hexane $(500 \mathrm{ml} \times 4)$, ethyl acetate $(500 \mathrm{ml} \times$ 4) and $n$-butanol $(500 \mathrm{ml} \times 3)$, successively. Each fraction was concentrated under reduced pressure to give $n$-hexane $(36.5 \mathrm{~g})$, ethyl acetate $(31.8 \mathrm{~g}), n$-butanol $(28.5 \mathrm{~g})$ and aqueous $(33.0 \mathrm{~g})$ residue.

About $30.8 \mathrm{~g}$ of the ethyl acetate fraction was subjected to VLC using $n$-hexane-ethyl acetate and ethyl acetate-methanol gradients to obtain seven group fractions: fraction E-1 (4.3 g, eluted with $n$-hexane-ethyl acetate 75:25), fraction E-2 (5.3 g, eluted with $n$-hexane-ethyl acetate 50:50), fraction E-3 (3.0 g, eluted with $n$-hexane-ethyl acetate 25:75), fraction E-4 (3.7 g, eluted with ethyl acetate), fraction E-5 (3.0 g, eluted with ethyl acetate-methanol 75:25), fraction E-6 (4.0 g, eluted with ethyl acetate- methanol 50:50) and fraction E-7 (6.0 g, eluted with methanol). About $500 \mathrm{mg}$ of fraction E-2 was submitted to HPLC to yield pure compounds $\mathbf{1}$ (8 mg), 2 (3 mg), 3 (9 
$\mathrm{mg}), 4(5 \mathrm{mg})$ and $5(9 \mathrm{mg})$. Fraction E-3 was subjected to silica gel column chromatography $(90.0 \mathrm{~g}, 50 \times$ $3 \mathrm{~cm})$ using dichloromethanemethanol $94: 6$ to afford pure compounds 6 (11 mg), 7 (15 mg) and $8(11 \mathrm{mg})$. About $2.0 \mathrm{~g}$ of fraction E6 was chromatographed over Sephadex LH-20 column (100 g, $60 \times$ $2 \mathrm{~cm}$ ) using methanol as an eluent to obtain two main subfractions A (500 $\mathrm{mg})$ and B $(1.0 \mathrm{~g})$. Repeated crystallization of subfraction E-6-A from methanol yielded pure compound $9(17 \mathrm{mg})$. The subfraction E-6-B was rechromatographed over RP-18 column $(100 \mathrm{~g}, 30 \times 1.5 \mathrm{~cm})$ using methanol-water $2: 3$ to afford pure compounds $\mathbf{1 0}(16 \mathrm{mg})$ and $\mathbf{1 1}$ (12 mg).

About $27.5 \mathrm{~g}$ of the n-butanol fraction was submitted to VLC using gradient dichloromethane-methanol to get four group fractions: fraction B-1 (4.0 g, eluted with dichloromethanemethanol 75:25), fraction B-2 (7.2 g, eluted with dichloromethanemethanol 50:50), fraction B-3 (8.8 g, eluted with dichloromethanemethanol 25:75) and fraction B-4 (6.0 $\mathrm{g}$, eluted with methanol). About $2.0 \mathrm{~g}$ of fraction B-2 was chromatographed on Sephadex LH-20 column (100 g, $60 \times 2 \mathrm{~cm}$ ) using methanol to give two main subfractions B-2-I (700 mg) and B-2-II (1.0 g). Rechromatography of the subfraction B-2-I on RP-18 column $(100 \mathrm{~g}, 30 \times 1.5 \mathrm{~cm})$ using methanol-water 30:70 afforded pure compounds $\mathbf{1 2}(18 \mathrm{mg}$ ) and $\mathbf{1 3}$ (12 $\mathrm{mg})$. Purification of the subfraction B-2-II on silica gel column (30 g, 30 $\times 1.5 \mathrm{~cm})$ using dichloromethanemethanol (70:30) yielded pure compound $14(21 \mathrm{mg})$. About $2 \mathrm{~g}$ of fraction B-3 was purified by Sephadex LH-20 column chromatography $(100 \mathrm{~g}, 60 \times 2 \mathrm{~cm})$ using methanol followed by rechromatography on RP-18 column $(100 \mathrm{~g}, 30 \times 1.5 \mathrm{~cm})$ using methanolwater 30:70 to give pure compounds $\mathbf{1 5}(26 \mathrm{mg})$ and $\mathbf{1 6}(13 \mathrm{mg})$.

Compound 1: Colourless prisms

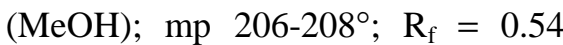
(solvent system I); UV $\lambda_{\max }(\mathrm{MeOH})$ nm: 229, 345. ${ }^{1} \mathrm{H}-\mathrm{NMR}(500 \mathrm{MHz}$, $\left.\mathrm{CDCl}_{3}\right): \delta 7.60(1 \mathrm{H}, \mathrm{d}, J=9.5 \mathrm{~Hz}, \mathrm{H}-$ 4), $6.91(1 \mathrm{H}, \mathrm{s}, \mathrm{H}-8), 6.84(1 \mathrm{H}, \mathrm{s}, \mathrm{H}-$ 5), $6.27(1 \mathrm{H}, \mathrm{d}, J=9.5 \mathrm{~Hz}, \mathrm{H}-3), 6.21$ $(1 \mathrm{H}, \mathrm{s}, 7-\mathrm{OH})$ and $3.95(3 \mathrm{H}, \mathrm{s}, 6-$ $\left.\mathrm{OCH}_{3}\right) . \quad{ }^{13} \mathrm{C}-\mathrm{NMR} \quad(125 \mathrm{MHz}$, $\mathrm{CDCl}_{3}$ ): $\delta 161.47$ (s, C-2), 150.22 (s, C-7), 149.69 (s, C-9), 144.01 (s, C-6), 143.31 (d, C-4), 113.36 (d, C-3), 111.47 (s, C-10), 107.48 (d, C-5), 103.17 (d, C-8), 56.39 (q, 6-Oㅡㅐ ${ }_{3}$ ). EI-MS $\mathrm{m} / \mathrm{z}$ (rel. int.\%): $192[\mathrm{M}]^{+}$ $\mathrm{C}_{10} \mathrm{H}_{8} \mathrm{O}_{4}$ (100), 177 (65), 164 (31), 149 (60), 129 (51), 121 (19), 111 (13), 97 (7), 83 (13), 69 (29), 57 (19), 55 (21), 44 (19).

Compound 2: Yellow powder; $\mathrm{R}_{\mathrm{f}}=$ 0.52 (solvent system I); UV $\lambda_{\max }$ $(\mathrm{MeOH}) \mathrm{nm}: 240,373 .{ }^{1} \mathrm{H}-\mathrm{NMR}(500$ $\left.\mathrm{MHz}, \mathrm{CDCl}_{3}\right): \delta 13.53\left(1 \mathrm{H}, \mathrm{s}, 2^{\prime}-\right.$ $\mathrm{OH}), 7.85(1 \mathrm{H}, \mathrm{d}, J=15.5 \mathrm{~Hz}, \mathrm{H}-\beta)$, $7.83\left(1 \mathrm{H}, \mathrm{d}, J=9.2 \mathrm{~Hz}, \mathrm{H}-6^{\prime}\right), 7.58$ $(2 \mathrm{H}, \mathrm{d}, J=8.8 \mathrm{~Hz}, \mathrm{H}-2 / 6), 7.46(1 \mathrm{H}$, d, $J=15.5 \mathrm{~Hz}, \mathrm{H}-\alpha), 6.96(1 \mathrm{H}, \mathrm{br} \mathrm{s}$, H-3'), 6.89 (2H, d, $J=8.8 \mathrm{~Hz}, \mathrm{H}-3 / 5)$, 
<smiles>COc1cc2ccc(=O)oc2cc1O</smiles>

Compound 1<smiles>COc1cc(/C=C/C(=O)O)ccc1O</smiles>

Compound 8<smiles>COc1ccc(C(=O)C=Cc2ccc(O)cc2)c(O)c1</smiles>

Compound 2<smiles>COc1cc(O)cc2c1C(=O)C(=Cc1ccc(O)c(O)c1)O2</smiles>

Compound 5

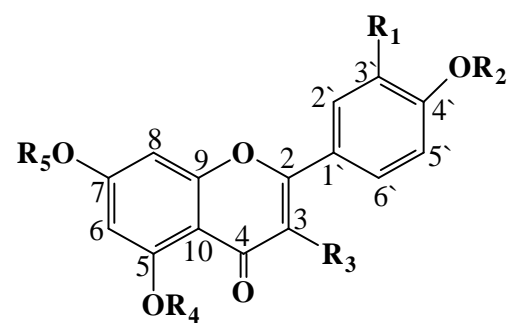

Compound $3 \mathrm{R}^{1}=\mathrm{OCH}_{3}, \mathrm{R}^{4}=\mathrm{CH}_{3}, \mathrm{R}^{2}=\mathrm{R}^{3}=\mathrm{R}^{5}=\mathrm{H}$

Compound $4 \mathrm{R}^{1}=\mathrm{OCH}_{3}, \mathrm{R}^{5}=\mathrm{CH}_{3}, \mathrm{R}^{2}=\mathrm{R}^{3}=\mathrm{R}^{4}=\mathrm{H}$

Compound $6 \mathrm{R}^{1}=\mathrm{R}^{2}=\mathrm{R}^{3}=\mathrm{R}^{4}=\mathrm{R}^{5}=\mathrm{H}$

Compound $7 \mathrm{R}^{1}=\mathrm{OH}, \mathrm{R}^{2}=\mathrm{R}^{3}=\mathrm{R}^{4}=\mathrm{R}^{5}=\mathrm{H}$

Compound $9 \mathrm{R}^{1}=\mathrm{OH}, \mathrm{R}^{3}=\mathrm{R}^{4}=\mathrm{R}^{5}=\mathrm{H}, \mathrm{R}^{2}=\mathrm{Glc}$

Compound $10 \mathrm{R}^{1}=\mathrm{OH}, \mathrm{R}^{2}=\mathrm{R}^{3}=\mathrm{R}^{4}=\mathrm{H}, \mathrm{R}^{5}=\mathrm{Glc}$

Compound $11 \mathrm{R}^{1}=\mathrm{OH}, \mathrm{R}^{2}=\mathrm{R}^{4}=\mathrm{R}^{5}=\mathrm{H}, \mathrm{R}^{3}=O$ Glc

Compound $12 \mathrm{R}^{1}=\mathrm{R}^{2}=\mathrm{R}^{3}=\mathrm{R}^{4}=\mathrm{H}, \mathrm{R}^{5}=\mathrm{Glc}\left(2^{\prime \prime} \rightarrow 1^{\prime \prime \prime}\right)$ rha

Compound $13 \mathrm{R}^{1}=\mathrm{R}^{2}=\mathrm{R}^{4}=\mathrm{R}^{5}=\mathrm{H}, \mathrm{R}^{3}=O$-Glc $\left(6^{\prime \prime} \rightarrow 1^{\prime \prime \prime}\right)$ rha

Compound $14 \mathrm{R}^{1}=\mathrm{OH}, \mathrm{R}^{2}=\mathrm{R}^{4}=\mathrm{R}^{5}=\mathrm{H}, \mathrm{R}^{3}=O$-Glc $\left(6^{\prime \prime} \rightarrow 1^{\prime \prime \prime}\right)$ rha

Compound $15 \mathrm{R}^{1}=\mathrm{R}^{2}=\mathrm{R}^{4}=\mathrm{R}^{5}=\mathrm{H}, \mathrm{R}^{3}=O-2^{\mathrm{G}}$-xylosylrutinose

Compound $16 \mathrm{R}^{1}=\mathrm{R}^{2}=\mathrm{R}^{4}=\mathrm{R}^{5}=\mathrm{H}, \mathrm{R}^{3}=O-2^{\mathrm{G}}$-glucosylrutinose

Fig. 1: Structures of the isolated compounds. 
$6.48\left(1 \mathrm{H}, \mathrm{dd}, J=9.2,1.6 \mathrm{~Hz}, \mathrm{H}-5^{\prime}\right)$, $3.86\left(3 \mathrm{H}, \mathrm{s}, 4^{\prime}-\mathrm{OCH}_{3}\right)$. Positive ESIMS $\mathrm{m} / \mathrm{z}$ (rel. int.\%): $271[\mathrm{M}+\mathrm{H}]^{+}$ $\mathrm{C}_{16} \mathrm{H}_{14} \mathrm{O}_{4}$ (100), 151 (15) and 147 (23).

Compound 3: Yellow powder; $\mathrm{R}_{\mathrm{f}}=$ 0.70 (solvent system II); UV $\lambda_{\max }$ $(\mathrm{MeOH}) \mathrm{nm}: 243,249$ sh, 268, 340; +NaOMe: 268, 275 sh, 329 sh, 403; $+\mathrm{AlCl}_{3}:$ 243, 249 sh, 269, 340; $+\mathrm{AlCl}_{3} / \mathrm{HCl}: 243,249$ sh, 269, 340; +NaOAc: $263, \quad 321, \quad 394$; $+\mathrm{NaOAc} / \mathrm{H}_{3} \mathrm{BO}_{3}: 268,347 .{ }^{1} \mathrm{H}-\mathrm{NMR}$ $\left(500 \mathrm{MHz}, \mathrm{CD}_{3} \mathrm{OD}\right): \delta 7.47(1 \mathrm{H}, \mathrm{dd}$, $\left.J=8.5,2.2 \mathrm{~Hz}, \mathrm{H}-6^{\prime}\right), 7.45(1 \mathrm{H}, \mathrm{d}, J$ $\left.=2.2 \mathrm{~Hz}, \mathrm{H}-2^{\prime}\right), 6.92(1 \mathrm{H}, \mathrm{d}, J=8.5$ $\left.\mathrm{Hz}, \mathrm{H}-5^{\prime}\right), 6.55(1 \mathrm{H}, \mathrm{s}, \mathrm{H}-3), 6.54$ $(1 \mathrm{H}, \mathrm{d}, J=2.2 \mathrm{~Hz}, \mathrm{H}-8), 6.41(1 \mathrm{H}, \mathrm{d}$, $J=2.2 \mathrm{~Hz}, \mathrm{H}-6), 3.95$ (3H, s, 3'$\left.\mathrm{OC}_{3}\right), \quad 3.88 \quad\left(3 \mathrm{H}, \quad\right.$ s, $\left.\quad 5-\mathrm{OC}_{3}\right)$. Positive-ion ESI-MS $\mathrm{m} / \mathrm{z}$ (rel. int.\%): $315[\mathrm{M}+\mathrm{H}]^{+} \mathrm{C}_{17} \mathrm{H}_{14} \mathrm{O}_{6}(100)$.

Compound 4: Yellow powder; $\mathrm{R}_{\mathrm{f}}=$ 0.65 (solvent system II); UV $\lambda_{\max }$ $(\mathrm{MeOH}) \mathrm{nm}: 245$ sh, 252, 267, 347; +NaOMe: 262, 292 sh, 402; $+\mathrm{AlCl}_{3}$ : 267 sh, 274, 296, 369 sh, 392; $+\mathrm{AlCl}_{3} / \mathrm{HCl}: 262,276,291$ sh, 355, 390; +NaOAc: 254, 263 sh, 288, 350, 406; +NaOAc/ $\mathrm{H}_{3} \mathrm{BO}_{3}: 250,267,348$. ${ }^{1} \mathrm{H}-\mathrm{NMR}\left(500 \mathrm{MHz}, \mathrm{CDCl}_{3}\right): \delta 12.79$ $(1 \mathrm{H}, \mathrm{s}, 5-\mathrm{OH}), 7.49(1 \mathrm{H}, \mathrm{dd}, J=7.5$, $\left.2.2 \mathrm{~Hz}, \mathrm{H}-6^{\prime}\right), 7.33(1 \mathrm{H}, \mathrm{d}, J=2.2$ $\left.\mathrm{Hz}, \mathrm{H}-2^{\prime}\right), 7.04(1 \mathrm{H}, \mathrm{d}, J=7.5 \mathrm{~Hz}, \mathrm{H}-$ 5'), $6.57(1 \mathrm{H}, \mathrm{s}, \mathrm{H}-3), 6.49(1 \mathrm{H}, \mathrm{d}, J=$ $2.2 \mathrm{~Hz}, \mathrm{H}-8), 6.38(1 \mathrm{H}, \mathrm{d}, J=2.2 \mathrm{~Hz}$, $\mathrm{H}-6), 4.01$ (3H, s, 3'-OCH $\left.{ }_{3}\right), 3.89$ $\left(3 \mathrm{H}\right.$, s, 7- $\left.-\mathrm{OCH}_{3}\right)$. Positive-ion ESI-
MS $m / z$ (rel. int.\%): $315[\mathrm{M}+\mathrm{H}]^{+}$ $\mathrm{C}_{17} \mathrm{H}_{14} \mathrm{O}_{6}(100)$.

Compound 5: Yellow powder; $\mathrm{R}_{\mathrm{f}}=$ 0.59 (solvent system II); UV $\lambda_{\max }$ $(\mathrm{MeOH}) \mathrm{nm}: 254 \mathrm{sh}, 270$ sh, 330 sh, 400; +NaOMe: 253, 273 sh, 383 sh, 402, 465; $+\mathrm{AlCl}_{3}: 259$ sh, 287, 342, 448; $+\mathrm{AlCl}_{3} / \mathrm{HCl}$ : 255 sh, 270 sh, 329 sh, 404; +NaOAc: 268, 316 sh, 384 sh, 420; $+\mathrm{NaOAc} / \mathrm{H}_{3} \mathrm{BO}_{3}$ : 264, 280, 346 sh, 435. ${ }^{1} \mathrm{H}-\mathrm{NMR}(500 \mathrm{MHz}$, DMSO- $\left.d_{6}\right): \delta 7.38(1 \mathrm{H}, \mathrm{d}, J=1.9 \mathrm{~Hz}$, H-2') $7.16(1 \mathrm{H}, \mathrm{dd}, J=8.2,1.9 \mathrm{~Hz}$, H-6'), $6.80\left(1 \mathrm{H}, \mathrm{d}, J=8.2 \mathrm{~Hz}, \mathrm{H}-5^{\prime}\right)$, $6.46(1 \mathrm{H}, \mathrm{s}, \mathrm{H}-10), 6.29(1 \mathrm{H}, \mathrm{d}, J=$ $1.6 \mathrm{~Hz}, \mathrm{H}-7), 6.14(1 \mathrm{H}, \mathrm{d}, J=1.6 \mathrm{~Hz}$, $\mathrm{H}-5), \quad 3.82\left(3 \mathrm{H}, \quad \mathrm{s}, 4-\mathrm{OCH}_{3}\right) .{ }^{13} \mathrm{C}-$ NMR (125 MHz, DMSO- $\left.d_{6}\right): \delta$ 178.40 (s, C-3), 168.40 (s, C-6), 167.76 (s, C-8), 159.28 (s, C-4), 147.57 (s, C-4'), 145.76 (s, C-2), 145.46 (s, C-3'), 124.02 (d, C-6'), 123.53 (s, C-1'), 117.57 (d, C-2'), 115.95 (d, C-5'), 110.01 (d, C-10), 102.86 (s, C-9), 94.66 (d, C-5), 91.30 (d, C-7), 55.76 (q, 4-Oㅡㅐ ${ }_{3}$ ). Positiveion ESI-MS m/z (rel. int.\%): 301 $[\mathrm{M}+\mathrm{H}]^{+} \mathrm{C}_{16} \mathrm{H}_{12} \mathrm{O}_{6}(100)$.

Compound 6: Yellow powder; $\mathrm{R}_{\mathrm{f}}=$ 0.58 (solvent system II); UV $\lambda_{\max }$ $(\mathrm{MeOH})$ nm: 268, 337; +NaOMe: 275, 392; + $\mathrm{AlCl}_{3}: 276,300,345,384$; $+\mathrm{AlCl}_{3} / \mathrm{HCl}: \quad 276, \quad 298, \quad 341, \quad 380$; +NaOAc: $\quad 275, \quad 300, \quad 375$; $+\mathrm{NaOAc} / \mathrm{H}_{3} \mathrm{BO}_{3}: 268,338 .{ }^{1} \mathrm{H}-\mathrm{NMR}$ $\left(500 \mathrm{MHz}, \mathrm{DMSO}-d_{6}\right): \delta 12.95(1 \mathrm{H}$, s, 5-OH), $7.91(2 \mathrm{H}, \mathrm{d}, J=8.8 \mathrm{~Hz}, \mathrm{H}-$ $\left.2^{\prime} / 6^{\prime}\right), 6.91(2 \mathrm{H}, \mathrm{d}, J=8.8 \mathrm{~Hz}, \mathrm{H}-$ $\left.3^{\prime} / 5^{\prime}\right), 6.76(1 \mathrm{H}, \mathrm{s}, \mathrm{H}-3), 6.46(1 \mathrm{H}, \mathrm{d}$, 
$J=1.9 \mathrm{~Hz}, \mathrm{H}-8), 6.18(1 \mathrm{H}, \mathrm{d}, J=1.9$ $\mathrm{Hz}, \quad \mathrm{H}-6) .{ }^{13} \mathrm{C}-\mathrm{NMR} \quad(125 \mathrm{MHz}$, DMSO- $d_{6}$ ): $\delta 181.69$ (s, C-4), 164.39 (s, C-2), 163.68 (s, C-7), 161.42 (s, C-5), 161.19 (s, C-9), 157.30 (s, C4'), 128.45 (d, C-2'/6'), 121.12 (s, C1'), 115.94 (d, C-3'/5'), 103.58 (s, C10), 102.79 (d, C-3), 98.87 (d, C-6), 93.98 (d, C-8). Positive-ion ESI-MS $\mathrm{m} / \mathrm{z} \quad$ (rel. int.\%): $271 \quad[\mathrm{M}+\mathrm{H}]^{+}$ $\mathrm{C}_{15} \mathrm{H}_{10} \mathrm{O}_{5}(100)$.

Compound 7: Yellow powder; $\mathrm{R}_{\mathrm{f}}=$ 0.51 (solvent system II); UV $\lambda_{\max }$ $(\mathrm{MeOH}) \mathrm{nm}: 242 \mathrm{sh}, 255,267,290$ sh, 350; +NaOMe: 267, 329, 402; $+\mathrm{AlCl}_{3}: 274,300$ sh, 328, 427; $+\mathrm{AlCl}_{3} / \mathrm{HCl}: 266 \mathrm{sh}, 276,294 \mathrm{sh}$, 355, 385; +NaOAc: 270, 326 sh, 384; $+\mathrm{NaOAc} / \mathrm{H}_{3} \mathrm{BO}_{3}: 260,300$ sh, 370 , 430 sh. ${ }^{1} \mathrm{H}-\mathrm{NMR}(500 \mathrm{MHz}$, DMSO$\left.d_{6}\right): \delta 12.97(1 \mathrm{H}, \mathrm{s}, 5-\mathrm{OH}), 7.40(1 \mathrm{H}$, $\left.\mathrm{dd}, J=8.2,2.2 \mathrm{~Hz}, \mathrm{H}-6^{\prime}\right), 7.39(1 \mathrm{H}$, $\left.\mathrm{d}, J=2.2 \mathrm{~Hz}, \mathrm{H}-2^{\prime}\right), 6.88(1 \mathrm{H}, \mathrm{d}, J=$ $\left.8.2 \mathrm{~Hz}, \mathrm{H}-5^{\prime}\right), 6.66(1 \mathrm{H}, \mathrm{s}, \mathrm{H}-3), 6.43$ $(1 \mathrm{H}, \mathrm{d}, J=2.2 \mathrm{~Hz}, \mathrm{H}-8), 6.18(1 \mathrm{H}, \mathrm{d}$, $J=2.2 \mathrm{~Hz}, \mathrm{H}-6)$. Positive-ion ESIMS $m / z$ (rel. int.\%): $287[\mathrm{M}+\mathrm{H}]^{+}$ $\mathrm{C}_{15} \mathrm{H}_{10} \mathrm{O}_{6}(100)$.

Compound 8: White needles $(\mathrm{MeOH}) ; \mathrm{mp} 169-171^{\circ} ; \mathrm{R}_{\mathrm{f}}=0.21$ (solvent system I); UV $\lambda_{\max }(\mathrm{MeOH})$ nm: 244, 296, 323. ${ }^{1}$ H-NMR (500 MHz, DMSO- $\left.d_{6}\right): \delta 12.19(1 \mathrm{H}, \mathrm{s}, 9-$ $\mathrm{COO} \underline{\mathrm{H}}), 9.17(1 \mathrm{H}, \mathrm{s}, 4-\mathrm{OH}), 7.43$ $(1 \mathrm{H}, \mathrm{d}, J=16.0 \mathrm{~Hz}, \mathrm{H}-7), 7.08(1 \mathrm{H}$, dd, $J=8.2,1.9 \mathrm{~Hz}, \mathrm{H}-6), 7.06(1 \mathrm{H}, \mathrm{d}$, $J=1.9 \mathrm{~Hz}, \mathrm{H}-2), 6.93(1 \mathrm{H}, \mathrm{d}, J=8.2$ $\mathrm{Hz}, \mathrm{H}-5), 6.23(1 \mathrm{H}, \mathrm{d}, J=16.0 \mathrm{~Hz}$, $\mathrm{H}-8), 3.79\left(3 \mathrm{H}, \mathrm{s}, 3-\mathrm{OC}_{3}\right)$. EI-MS $m / z$ (rel. int.\%): $194[\mathrm{M}]^{+} \mathrm{C}_{10} \mathrm{H}_{10} \mathrm{O}_{4}$ (100), 179 (42), 161 (7), 151 (10), 149 (9), 133 (31), 123 (7), 117 (5), 105 (7), 95 (5), 89 (6), 77 (10), 67 (3), 51 (4), 43 (3), 40 (3).

Compound 9: Yellow powder; $\mathrm{R}_{\mathrm{f}}=$ 0.60 (solvent system III); UV $\lambda_{\max }$ $(\mathrm{MeOH}) \mathrm{nm}: 250 \mathrm{sh}, 268,291 \mathrm{sh}$, 340; +NaOMe: 270, 303 sh, 361; $+\mathrm{AlCl}_{3}: 267$ sh, 273, 296, 362, 390; $+\mathrm{AlCl}_{3} / \mathrm{HCl}: 264$ sh, 276, 295, 351, 382; +NaOAc: 276, 323, 366; $+\mathrm{NaOAc} / \mathrm{H}_{3} \mathrm{BO}_{3}: 253$ sh, 268, 347. ${ }^{1} \mathrm{H}-\mathrm{NMR}\left(500 \mathrm{MHz}\right.$, DMSO- $\left.d_{6}\right): \delta$ $12.89(1 \mathrm{H}, \mathrm{s}, 5-\mathrm{OH}), 7.50(1 \mathrm{H}, \mathrm{dd}, J$ $\left.=8.5,2.2 \mathrm{~Hz}, \mathrm{H}-6^{\prime}\right), 7.49(1 \mathrm{H}$, br s, $\left.\mathrm{H}-2^{\prime}\right), 7.23\left(1 \mathrm{H}, \mathrm{d}, J=8.5 \mathrm{~Hz}, \mathrm{H}-5^{\prime}\right)$, $6.80(1 \mathrm{H}, \mathrm{s}, \mathrm{H}-3), 6.49(1 \mathrm{H}, \mathrm{d}, J=1.9$ $\mathrm{Hz}, \mathrm{H}-8), 6.19(1 \mathrm{H}, \mathrm{d}, J=1.9 \mathrm{~Hz}, \mathrm{H}-$ 6), $4.89\left(1 \mathrm{H}, \mathrm{d}, J=7.3 \mathrm{~Hz}, \mathrm{H}-1^{\prime \prime}\right)$, 3.73-3.19 (m, sugar protons). ${ }^{13} \mathrm{C}-$ NMR $\left(125 \mathrm{MHz}\right.$, DMSO- $\left.d_{6}\right): \delta$ 181.79 (s, C-4), 164.45 (s, C-2), 163.20 (s, C-7), 161.47 (s, C-5), 157.39 (s, C-9), 148.58 (s, C-4'), 146.98 (s, C-3'), 124.76 (s, C-1'), 118.54 (d, C-6'), 116.03 (d, C-5'), 113.64 (d, C-2'), 104.00 (d, C-3), 103.80 (s, C-10), 101.24 (d, C-1"), 99.00 (d, C-6), 94.09 (d, C-8), 77.33 (d, C-5"), 75.89 (d, C-3"), 73.27 (d, C-2"), 69.80 (d, C-4"), 60.74 (t, C6"). Positive-ion ESI-MS $\mathrm{m} / \mathrm{z}$ (rel. int.\%): $449[\mathrm{M}+\mathrm{H}]^{+} \mathrm{C}_{21} \mathrm{H}_{20} \mathrm{O}_{11}(100)$, $287[\mathrm{M}-\mathrm{Glc}+\mathrm{H}]^{+}(23)$.

Compound 10: Yellow powder; $\mathrm{R}_{\mathrm{f}}=$ 0.57 (solvent system III); UV $\lambda_{\max }$ $(\mathrm{MeOH}) \mathrm{nm}: 255,268,291$ sh, 349; +NaOMe: 263, 300 sh, 395; $+\mathrm{AlCl}_{3}$ : 
274, 298 sh, 329, 433; + $\mathrm{AlCl}_{3} / \mathrm{HCl}$ : 273, 294 sh, 358, 388; +NaOAc: 259, 266, 367 sh, 404; $+\mathrm{NaOAc} / \mathrm{H}_{3} \mathrm{BO}_{3}$ : 259, 373. ${ }^{1} \mathrm{H}-\mathrm{NMR} \quad(500 \mathrm{MHz}$, DMSO- $\left.d_{6}\right): \delta 12.98(1 \mathrm{H}, \mathrm{s}, 5-\mathrm{O} \underline{\mathrm{H}})$, $7.44\left(1 \mathrm{H}, \mathrm{dd}, J=8.2,2.2 \mathrm{~Hz}, \mathrm{H}-6^{\prime}\right)$, $7.41\left(1 \mathrm{H}, \mathrm{d}, J=2.2 \mathrm{~Hz}, \mathrm{H}-2^{\prime}\right), 6.89$ $\left(1 \mathrm{H}, \mathrm{d}, J=8.2 \mathrm{~Hz}, \mathrm{H}-5^{\prime}\right), 6.78(1 \mathrm{H}, \mathrm{d}$, $J=2.2 \mathrm{~Hz}, \mathrm{H}-8), 6.74(1 \mathrm{H}, \mathrm{s}, \mathrm{H}-3)$, $6.43(1 \mathrm{H}, \mathrm{d}, J=2.2 \mathrm{~Hz}, \mathrm{H}-6), 5.08$ $\left(1 \mathrm{H}, \mathrm{d}, J=7.6 \mathrm{~Hz}, \mathrm{H}-1^{\prime \prime}\right), 3.71-3.15$ (m, sugar protons). ${ }^{13} \mathrm{C}-\mathrm{NMR}$ (125 MHz, DMSO- $d_{6}$ ): $\delta 181.90$ (s, C-4), 164.47 (s, C-2), 162.94 (s, C-7), 161.13 (s, C-5), 156.94 (s, C-9), 149.92 (s, C-4'), 145.78 (s, C-3'), 121.38 (s, C-1'), 119.18 (d, C-6'), 116.00 (d, C-5'), 113.56 (d, C-2'), 105.34 (s, C-10), 103.17 (d, C-3), 99.86 (d, C-1"), 99.53 (d, C-6), 94.71 (d, C-8), 77.16 (d, C-5"), 76.39 (d, C3"), 73.12 (d, C-2"), 69.53 (d, C-4"), 60.60 (t, C-6"). Positive-ion ESI-MS $\mathrm{m} / \mathrm{z} \quad$ (rel. int.\%): $449 \quad[\mathrm{M}+\mathrm{H}]^{+}$ $\mathrm{C}_{21} \mathrm{H}_{20} \mathrm{O}_{11}$ (100), $287 \quad[\mathrm{M}-\mathrm{Glc}+\mathrm{H}]^{+}$ (35).

Compound 11: Yellow powder; $\mathrm{R}_{\mathrm{f}}=$ 0.55 (solvent system III); UV $\lambda_{\max }$ $(\mathrm{MeOH}) \mathrm{nm}: 257,266 \mathrm{sh}, 296 \mathrm{sh}$, 357; +NaOMe: 271, 327, 400; $+\mathrm{AlCl}_{3}: 275,304$ sh, 332, 437; $+\mathrm{AlCl}_{3} / \mathrm{HCl}: 271,303 \mathrm{sh}, 353,402$; +NaOAc: $273, \quad 325$ sh, 379 ; $+\mathrm{NaOAc} / \mathrm{H}_{3} \mathrm{BO}_{3}$ : 261, 298 sh, 374. ${ }^{1} \mathrm{H}-\mathrm{NMR}\left(500 \mathrm{MHz}, \mathrm{DMSO}-d_{6}\right): \delta$ $12.63(1 \mathrm{H}, \mathrm{s}, 5-\mathrm{OH}), 7.57(1 \mathrm{H}, \mathrm{dd}, J$ $\left.=8.8,2.2 \mathrm{~Hz}, \mathrm{H}-6^{\prime}\right), 7.56(1 \mathrm{H}$, br s, H-2'), 6.83 (1H, d, $\left.J=8.8 \mathrm{~Hz}, \mathrm{H}-5^{\prime}\right)$, $6.40(1 \mathrm{H}, \mathrm{d}, \mathrm{br} \mathrm{s}, \mathrm{H}-8), 6.19(1 \mathrm{H}, \mathrm{d}$, br s, H-6), 5.46 (1H, d, $J=7.3 \mathrm{~Hz}, \mathrm{H}-$ $\left.1^{\prime \prime}\right)$, 3.58-3.08 (m, sugar protons). Positive-ion ESI-MS $m / z$ (rel. int.\%): $465[\mathrm{M}+\mathrm{H}]^{+} \mathrm{C}_{21} \mathrm{H}_{20} \mathrm{O}_{12}$ (100), 303 $[\mathrm{M}-\mathrm{Glc}+\mathrm{H}]^{+}(62)$.

Compound 12: Yellowish white powder; $\mathrm{R}_{\mathrm{f}}=0.32$ (solvent system III); UV $\lambda_{\max }(\mathrm{MeOH}) \mathrm{nm}: 267,339$; + NaOMe: 245 sh, 266, 392; $+\mathrm{AlCl}_{3}$ : 274, 300, 348, 388; $+\mathrm{AlCl}_{3} / \mathrm{HCl}: 275$, 299, 340, 384; +NaOAc: 268, 354, $393 ;+\mathrm{NaOAc} / \mathrm{H}_{3} \mathrm{BO}_{3}: 268,347 .{ }^{1} \mathrm{H}-$ NMR (500 MHz, DMSO- $d_{6}$ ): $\delta 12.96$ (1H, s, 5- $-\underline{\mathrm{H}}), 7.92(2 \mathrm{H}, \mathrm{d}, J=8.8$ $\left.\mathrm{Hz}, \mathrm{H}-2^{\prime} / 6^{\prime}\right), 6.93(2 \mathrm{H}, \mathrm{d}, J=8.8 \mathrm{~Hz}$, H-3'/5'), $6.85(1 \mathrm{H}, \mathrm{s}, \mathrm{H}-3), 6.78(1 \mathrm{H}$, d, $J=1.9 \mathrm{~Hz}, \mathrm{H}-8), 6.36(1 \mathrm{H}, \mathrm{d}, J=$ $1.9 \mathrm{~Hz}, \mathrm{H}-6), 5.23(1 \mathrm{H}, \mathrm{d}, J=7.3 \mathrm{~Hz}$, $\left.\mathrm{H}-1^{\prime \prime}\right), 5.12$ (1H, s, H-1"'), 5.37-3.21 (m, sugar protons), $1.20(3 \mathrm{H}, \mathrm{d}, J=$ $\left.6.0 \mathrm{~Hz} \mathrm{H}-66^{\prime \prime \prime}\right) .{ }^{13} \mathrm{C}-\mathrm{NMR}(125 \mathrm{MHz}$, DMSO- $\left.d_{6}\right): \delta 182.05$ (C-4), 164.34 (C-2), 162.59 (C-7), 161.43 (C-5), 161.17 (C-4'), 157.04 (C-9), 128.65 $\left(\mathrm{C}-2^{\prime} / 6^{\prime}\right), 121.05\left(\mathrm{C}-1^{\prime}\right), 116.10$ (C3'/5'), 105.49 (C-10), 103.24 (C-3), 100.54 (C-1"'), 99.38 (C-6), 97.85 (C-1"), 94.57 (C-8), 77.28 (C-5"), 77.07 (C-2"), 76.35 (C-3"), 71.93 (C$\left.4^{\prime \prime \prime}\right), 70.55$ (C-3"'), 70.47 (C-2"'), 69.70 (C-4"), 68.43 (C-5"'), 60.54 (C-6"), 18.15 (C-6"'). Positive-ion ESI-MS $m / z$ (rel. int.\%): $579[\mathrm{M}+\mathrm{H}]^{+}$ $\mathrm{C}_{27} \mathrm{H}_{30} \mathrm{O}_{14} \quad$ (100), 271 [M-Rha$\mathrm{Glc}+\mathrm{H}]^{+}(20)$.

Compound 13: Yellow powder; $\mathrm{R}_{\mathrm{f}}=$ 0.28 (solvent system III); UV $\lambda_{\max }$ $(\mathrm{MeOH}) \mathrm{nm}: 258$ sh, 266, 298, 347; +NaOMe: 275, 325, 401; $+\mathrm{AlCl}_{3}: 257$ 
sh, 275, 304, 354, 400; $+\mathrm{AlCl}_{3} / \mathrm{HCl}$ : 257 sh, 276, 303, 349, 398; +NaOAc: $274,316,390 ;+\mathrm{NaOAc} / \mathrm{H}_{3} \mathrm{BO}_{3}: 266$, 298 sh, 352. ${ }^{1} \mathrm{H}-\mathrm{NMR}(500 \mathrm{MHz}$, DMSO- $\left.d_{6}\right): \delta 12.52(1 \mathrm{H}, \mathrm{s}, 5-\mathrm{O} \underline{\mathrm{H}})$, $7.96\left(2 \mathrm{H}, \mathrm{d}, J=8.8 \mathrm{~Hz}, \mathrm{H}-2^{\prime} / 6^{\prime}\right), 6.86$ $\left(2 \mathrm{H}, \mathrm{d}, J=8.8 \mathrm{~Hz}, \mathrm{H}-3^{\prime} / 5^{\prime}\right), 6.34(1 \mathrm{H}$, $\mathrm{d}, J=1.5 \mathrm{~Hz}, \mathrm{H}-8), 6.14(1 \mathrm{H}, \mathrm{d}, J=$ $1.5 \mathrm{~Hz}, \mathrm{H}-6), 5.28(1 \mathrm{H}, \mathrm{d}, J=7.6 \mathrm{~Hz}$, H-1"), 4.37 (1H, s, H-1"'), 3.88-3.05 (m, sugar protons), $1.20(3 \mathrm{H}, \mathrm{d}, J=$ $\left.6.0 \mathrm{~Hz}, \mathrm{H}-6^{\prime \prime \prime}\right)$. Positive-ion ESI-MS $\mathrm{m} / \mathrm{z} \quad$ (rel. int.\%): $595 \quad[\mathrm{M}+\mathrm{H}]^{+}$ $\mathrm{C}_{27} \mathrm{H}_{30} \mathrm{O}_{15}$ (100), 449 [M-Rha+H] $]^{+}$ (43), 287 [M-Rha-Glc+H] ${ }^{+}$(45).

Compound 14: Yellow powder; $\mathrm{R}_{\mathrm{f}}=$ 0.21 (solvent system III); UV $\lambda_{\max }$ $(\mathrm{MeOH}) \mathrm{nm}:$ 256, $266 \mathrm{sh}, 299 \mathrm{sh}$, 357; +NaOMe: 272, 327, 400; $+\mathrm{AlCl}_{3}:$ 275, 304 sh, 335, 433; $+\mathrm{AlCl}_{3} / \mathrm{HCl}: 273,300$ sh, 355, 404; +NaOAc: $272, \quad 325$ sh, 380 ; $+\mathrm{NaOAc} / \mathrm{H}_{3} \mathrm{BO}_{3}: 260,300$ sh, 375 . ${ }^{1} \mathrm{H}-\mathrm{NMR}\left(500 \mathrm{MHz}\right.$, DMSO- $\left.d_{6}\right)$ : $\delta$ $12.59(1 \mathrm{H}, \mathrm{s}, 5-\mathrm{OH}), 7.53(1 \mathrm{H}, \mathrm{dd}, J$ $\left.=8.5,2.2 \mathrm{~Hz}, \mathrm{H}-6^{\prime}\right), 7.52(1 \mathrm{H}$, br s H$\left.2^{\prime}\right), 6.83\left(1 \mathrm{H}, \mathrm{d}, J=8.5 \mathrm{~Hz}, \mathrm{H}-5^{\prime}\right)$, $6.38(1 \mathrm{H}, \mathrm{d}, J=2.2 \mathrm{~Hz}, \mathrm{H}-8), 6.19$ $(1 \mathrm{H}, \mathrm{d}, J=2.2 \mathrm{~Hz}, \mathrm{H}-6), 5.34(1 \mathrm{H}, \mathrm{d}$, $\left.J=7.6 \mathrm{~Hz}, \mathrm{H}-1^{\prime \prime}\right), 4.37(1 \mathrm{H}, \mathrm{d}, J=0.6$ $\left.\mathrm{Hz}, \mathrm{H}-1^{\prime \prime \prime}\right), 0.98(3 \mathrm{H}, \mathrm{d}, J=6.3 \mathrm{~Hz}$, H-6"'), 5.33-3.05 (m, sugar protons). ${ }^{13} \mathrm{C}-\mathrm{NMR}\left(125 \mathrm{MHz}\right.$, DMSO- $\left.d_{6}\right): \delta$ 177.44 (s, C-4), 164.14 (s, C-7), 161.28 (s, C-5), 156.69 (s, C-9), 156.49 (s, C-2), 148.47 (s, C-4'), 144.81 (s, C-3'), 133.36 (s, C-3), 121.66 (d, C-6'), 121.25 (s, C-1'), 116.34 (d, C-5'), 115.30 (d, C-2'),
104.04 (s, C-10), 101.24 (d, C-1"), 100.81 (d, C-1'"'), 98.75 (d, C-6), 93.66 (d, C-8), 76.50 (d, C-5"), 75.96 (d, C-3"), 74.13 (d, C-2"), 71.90 (d, C-4"') 70.61 (d, C-3"') 70.44 (d, C$\left.2^{\prime \prime \prime}\right), 70.06$ (d, C-4"), 68.31 (d, C5"'), 67.06 (t, C-6"), 17.80 (q, C-6"'). Positive-ion ESI-MS $m / z$ (rel. int.\%): $611[\mathrm{M}+\mathrm{H}]^{+} \mathrm{C}_{27} \mathrm{H}_{30} \mathrm{O}_{16}$ (100), 465 $[\mathrm{M}-\mathrm{Rha}+\mathrm{H}]^{+} \quad(25), \quad 303 \quad$ [M-Rha$\mathrm{Glc}+\mathrm{H}]^{+}(35)$.

Compound 15: Yellowish brown powder; $\mathrm{R}_{\mathrm{f}}=0.84$ (solvent system IV); UV $\lambda_{\max }(\mathrm{MeOH}) \mathrm{nm}: 258 \mathrm{sh}$, 266, 295, 345; +NaOMe: 275, 325, 398; $+\mathrm{AlCl}_{3}: 257 \mathrm{sh}, 274,304,350$, 395; $+\mathrm{AlCl}_{3} / \mathrm{HCl}: 256 \mathrm{sh}, 275,303$, 349, 394; +NaOAc: 275, 315, 390; $+\mathrm{NaOAc} / \mathrm{H}_{3} \mathrm{BO}_{3}: 265,298$ sh, 348. ${ }^{1} \mathrm{H}-\mathrm{NMR}\left(500 \mathrm{MHz}\right.$, DMSO- $\left.d_{6}\right): \delta$ $12.62(1 \mathrm{H}, \mathrm{s}, 5-\mathrm{O} \underline{\mathrm{H}}), 8.01(2 \mathrm{H}, \mathrm{d}, J=$ $\left.8.8 \mathrm{~Hz}, \mathrm{H}-2^{\prime} / 6^{\prime}\right), 6.88(2 \mathrm{H}, \mathrm{d}, J=8.8$ $\left.\mathrm{Hz}, \mathrm{H}-3^{\prime} / 5^{\prime}\right), 6.36(1 \mathrm{H}$, br s, H-8), $6.16(1 \mathrm{H}$, br s, H-6), $5.57(1 \mathrm{H}, \mathrm{d}, J=$ $7.3 \mathrm{~Hz}, 3-\mathrm{Glc} \mathrm{H}-1), 4.59(1 \mathrm{H}, \mathrm{d}, J=$ $7.3 \mathrm{~Hz}, 2$ "-Xyl H-1), 4.34 (1H, s, Rha $\mathrm{H}-1$ ), 3.74-3.03 (m, sugar protons), $0.94\left(3 \mathrm{H}, \mathrm{d}, J=6.0 \mathrm{~Hz}, \mathrm{H}-6^{\prime \prime \prime}\right) .{ }^{13} \mathrm{C}-$ NMR (125 MHz, DMSO- $\left.d_{6}\right)$ : see Table 1. Positive-ion ESI-MS $\mathrm{m} / \mathrm{z}$ (rel. int.\%): $727[\mathrm{M}+\mathrm{H}]^{+} \mathrm{C}_{32} \mathrm{H}_{38} \mathrm{O}_{19}$ (100), $595[\mathrm{M}-\mathrm{Xyl}+\mathrm{H}]^{+}$(30), 449 [M$\mathrm{Xyl}-\mathrm{Rha}+\mathrm{H}]^{+}$(28), 287 [M-Xyl-Rha$\mathrm{Glc}+\mathrm{H}]^{+}(55)$.

Compound 16: Yellowish brown powder; $\mathrm{R}_{\mathrm{f}}=0.82$ (solvent system IV); UV $\lambda_{\max }(\mathrm{MeOH}) \mathrm{nm}: 266,296$, 346 ; +NaOMe: 274, 325, 399; $+\mathrm{AlCl}_{3}: 257$ sh, 275, 302, 354, 398; 
Table 1: ${ }^{13} \mathrm{C}-\mathrm{NMR}$ data for compounds 15 and $16(125 \mathrm{MHz}$ in DMSO- $d_{6}$ ).

\begin{tabular}{|c|c|c|}
\hline Carbon & Compd. 15 & Compd. 16 \\
\hline \multicolumn{3}{|l|}{ Aglycone } \\
\hline 2 & 155.93 & 155.50 \\
\hline 3 & 132.87 & 132.40 \\
\hline 4 & 177.41 & 176.65 \\
\hline 5 & 161.34 & 161.06 \\
\hline 6 & 99.04 & 100.42 \\
\hline 7 & 165.01 & 165.17 \\
\hline 8 & 93.94 & 94.25 \\
\hline 9 & 156.62 & 156.76 \\
\hline 10 & 103.72 & 103.74 \\
\hline $1^{\prime}$ & 121.06 & 121.04 \\
\hline $2^{\prime}, 6^{\prime}$ & 131.09 & 130.80 \\
\hline $4^{\prime}$ & 160.04 & 159.84 \\
\hline $3^{\prime}, 5^{\prime}$ & 115.33 & 115.18 \\
\hline \multicolumn{3}{|l|}{$\begin{array}{l}\text { Sugars } \\
\text { 3-Glc }\end{array}$} \\
\hline $1^{\prime \prime}$ & 98.33 & 98.33 \\
\hline $2^{\prime \prime}$ & 81.66 & 81.93 \\
\hline $3^{\prime \prime}$ & 76.83 & $76.51^{\mathrm{a}}$ \\
\hline $4^{\prime \prime}$ & 69.65 & 69.51 \\
\hline $5^{\prime \prime}$ & 75.84 & $75.47^{\mathrm{a}}$ \\
\hline $6^{\prime \prime}$ & 66.23 & 66.11 \\
\hline \multicolumn{3}{|c|}{$2^{\prime \prime}$-Glucose } \\
\hline 1 & & 103.74 \\
\hline 2 & & 74.17 \\
\hline 3 & & $76.33^{b}$ \\
\hline 4 & & 70.53 \\
\hline 5 & & $77.03^{b}$ \\
\hline 6 & & 60.80 \\
\hline \multicolumn{3}{|c|}{$2^{\prime \prime}$-Xylose } \\
\hline 1 & 104.49 & \\
\hline 2 & 73.88 & \\
\hline 3 & 76.16 & \\
\hline 4 & 69.54 & \\
\hline 5 & 65.76 & \\
\hline \multicolumn{3}{|c|}{ 6"-Rhamnose } \\
\hline 1 & 100.54 & 100.42 \\
\hline 2 & $70.44^{\mathrm{a}}$ & $70.26^{\mathrm{c}}$ \\
\hline 3 & $70.71^{a}$ & $69.66^{\mathrm{c}}$ \\
\hline 4 & 71.96 & 71.80 \\
\hline 5 & 68.34 & 68.18 \\
\hline 6 & 17.78 & 17.66 \\
\hline
\end{tabular}

Interchangeable assignments within the spectrum
$+\mathrm{AlCl}_{3} / \mathrm{HCl}: 256$ sh, 274, 300, 349, 396; +NaOAc: 275, 316, 390; $+\mathrm{NaOAc} / \mathrm{H}_{3} \mathrm{BO}_{3}$ : 266, 298 sh, 347. ${ }^{1} \mathrm{H}-\mathrm{NMR}$ (500 MHz, DMSO- $\left.d_{6}\right): \delta$ $12.56(1 \mathrm{H}, \mathrm{s}, 5-\mathrm{OH}), 7.94(2 \mathrm{H}, \mathrm{d}, J=$ $\left.8.8 \mathrm{~Hz}, \mathrm{H}-2^{\prime} / 6^{\prime}\right), 6.87$ (2H, d, $J=8.8$ $\left.\mathrm{Hz}, \mathrm{H}-3^{\prime} / 5^{\prime}\right), 6.19$ (1H, br s, H-8), $6.00(1 \mathrm{H}$, br s, H-6), $5.50(1 \mathrm{H}, \mathrm{d}, J=$ $7.0 \mathrm{~Hz}, 3-\mathrm{Glc} \mathrm{H}-1), 4.58$ (1H, d, $J=$ $7.6 \mathrm{~Hz}, 2^{\prime \prime}$-Glc H-1), 4.29 (1H, br s, Rha H-1), 5.42-3.04 (m, sugar protons), $0.95(3 \mathrm{H}, \mathrm{d}, J=6.3 \mathrm{~Hz}, \mathrm{H}-$ $\left.6^{\prime \prime \prime}\right) .{ }^{13} \mathrm{C}-\mathrm{NMR}$ (125 MHz, DMSO$\left.d_{6}\right)$ : see Table 1 . Positive-ion ESI-MS $m / z \quad$ (rel. int.\%): $757 \quad[\mathrm{M}+\mathrm{H}]^{+}$ $\mathrm{C}_{33} \mathrm{H}_{40} \mathrm{O}_{20}$ (100), $595 \quad[\mathrm{M}-\mathrm{Glc}+\mathrm{H}]^{+}$ (15), 449 [M-Glc-Rha+H $]^{+}$(20), 287 $[\mathrm{M}-\mathrm{Glc}-\mathrm{Rha}-\mathrm{Glc}+\mathrm{H}]^{+}(40)$.

\section{Acid hydrolysis ${ }^{31}$}

A solution of the isolated glycoside (5 $\mathrm{mg}$ in $10 \mathrm{ml}$ methanol) was treated with $3 \% \mathrm{H}_{2} \mathrm{SO}_{4}(1.5 \mathrm{ml})$ and heated at $100{ }^{\circ} \mathrm{C}$ for $1 \mathrm{hr}$. The aglycone was extracted with EtOAc, concentrated under reduced pressure, purified on Sephadex LH-20 column using methanol and identified by coTLC with an authentic sample using solvent system II. The sugars in the aqueous layer were identified by coTLC with authentic materials using solvent system V.

\section{Acid hydrolysis and GC analysis ${ }^{32}$}

A solution of compound $\mathbf{1 5}$ (5 $\mathrm{mg}$ in $25 \mathrm{ml}$ methanol) was treated with $3 \mathrm{~N} \mathrm{HCl}(15 \mathrm{ml})$ and stirred at $80^{\circ}$ for about $5 \mathrm{~h}$. Upon drying with a flow of nitrogen, the residue was dissolved in (-)-2-butanol (0.5 $\mathrm{ml})$ and one drop 
of trifluoroacetic acid. The solution was transferred to an ampoule which was sealed and heated at $130^{\circ}$ in an oven overnight until complete butanolysis. After being taken to dryness, the resulting residue was reacted with hexamethyldisilazane / chlorotrimethylsilane / pyridine $(1: 1: 5,0.1 \mathrm{ml})$ for $30 \mathrm{~min}$ at room temperature. The solution was then centrifuged and the supernatant $(1 \mu \mathrm{l})$ was analysed by an Agilent 6850 gas chromatograph. A temperature gradient from $135^{\circ}$ to $200^{\circ}$ at $1 \% \mathrm{~min}$ was applied. The injection volume was $1 \mu \mathrm{l}$. The carrier gas used was nitrogen with a split ratio: 50:1 and 1 $\mathrm{ml} / \mathrm{min}$ constant flow rate. The injection port and detector temperatures were set at $200^{\circ}$ and $220^{\circ}, \quad$ respectively. For the hydrolysate, four peaks were detected at $42.9,48.6,27.0$ and $31.7 \mathrm{~min}$. Authentic standards prepared in a similar manner from commercially available L-glucose, D-glucose and D-xylose, gave peaks at: 42.9 and 48.6 min for D-glucose; 41.7 and 48.4 min for L-glucose; 27.0 and $31.7 \mathrm{~min}$ for D-xylose.

\section{Antioxidant activity ${ }^{33}$}

The antioxidant activity was quantified by the decrease in the absorption of each of the isolated compounds or soluble fractions in $118 \times 10^{-5} \%$ DPPH solution (final concentration of the sample in the cuvette was $20 \mu \mathrm{M}$ for pure compounds and 0.5 and $1.0 \mathrm{mg} / \mathrm{ml}$ for soluble fractions) monitored at 517 $\mathrm{nm}$ using a spectrophotometer. The absorbance of DPPH in methanol (with or without compounds) was measured after $2 \mathrm{~min}$. The antioxidant activity of each compound was measured in relation to propyl gallate (a known synthetic antioxidant) set as $100 \%$ antioxidant activity. Determinations were performed in triplicate. The antioxidant activity was calculated using the following equation: ${ }^{34}$

Antioxidant activity $=100 \mathrm{x}$

$$
\left(1-\frac{\text { absorbance with compound }}{\text { absorbance of the blank }}\right)
$$

\section{Cytotoxic activity $^{35}$}

L5178Y mouse lymphoma cells were grown in Eagle's minimal essential medium supplemented with $10 \%$ horse serum in roller tube culture. For the dose-response experiments, $5 \mathrm{ml}$ cultures were initiated by inoculation of $5 \times 10^{3}$ cells $/ \mathrm{ml}$ and incubated at $37^{\circ}$ for $72 \mathrm{~h}$. Controls showed a population doubling time of $10.5 \mathrm{~h}$. Cell growth was determined by a cell count with a Cytocomp counter (128-channel counter, system Michaelis, Mainz, Germany) incorporating a 32-channel size-distribution plotter.

\section{$\alpha$-amylase inhibitory activity ${ }^{36}$}

The method is based on assay of $\alpha$-amylase by EnzCheck ${ }^{\circledR}$ Amylase Assay Kit (E-11954). The provided stock solution of DQ starch and $\alpha$ amylase enzyme were diluted with the reaction buffer (pH 6.9) according to 
the reported protocol. $^{36}$ To the microplate wells, $50 \mu \mathrm{g} / 10 \mu \mathrm{l}$ of the tested compound in DMSO, $50 \mu \mathrm{l}$ of the diluted enzyme and $40 \mu \mathrm{l}$ of the reaction buffer were added and allowed to stand for $5 \mathrm{~min}$ at room temperature then $100 \mu \mathrm{l}$ of DQ starch was added. The fluorescence intensity of the digestion products from the DQ starch (with or without compounds) was measured using a kinetic assay program in the Tecan Genios microplate reader at $\lambda_{\max } 485 \pm 10 \mathrm{~nm}$ starting from zero min to $60 \mathrm{~min}$ at 10 min intervals. All determinations were performed in triplicate. The $\alpha$ amylase inhibitory activity of each test compound was measured in relation to acarbose (Ac) set as $100 \%$ $\alpha$-amylase inhibitory activity. The percentage of $\alpha$-amylase activity and $\alpha$-amylase inhibition were calculated using the following equations:

$\% \alpha-$ amylase activity $=100 \mathrm{x}$

$$
\left(\mathrm{F}_{\mathrm{s}}^{60}-\mathrm{F}_{\mathrm{s}}^{0}\right) /\left(\mathrm{F}_{\mathrm{c}}^{60}-\mathrm{F}_{\mathrm{c}}^{0}\right)
$$

where $\mathrm{F}_{\mathrm{s}}^{60}$ : Fluorescence with the sample at $60 \mathrm{~min}, \mathrm{~F}_{\mathrm{s}}{ }^{0}$ : Fluorescence with the sample at $0 \mathrm{~min}, \mathrm{~F}_{\mathrm{c}}^{60}$ : Fluorescence of the control at $60 \mathrm{~min}$, $\mathrm{F}_{\mathrm{c}}^{0}$ : Fluorescence of the control at 0 min.

$\% \alpha$-amylase inhibition $=$ $100-\% \alpha$-amylase activity

\section{RESULTS AND DISCUSSION}

The methanolic extract of $C$. alopecuroides Rottb. inflorescences was successively partitioned between water and $n$-hexane, ethyl acetate and $n$-butanol. The ethyl acetate and $n$ butanol soluble fractions through series of different chromatographic fractionation techniques, afforded sixteen phenolic compounds 1-16.

Compound $\mathbf{1}$ was assigned a molecular formula of $\mathrm{C}_{10} \mathrm{H}_{8} \mathrm{O}_{4}$ by EIMS analysis that appeared $[\mathrm{M}]^{+}$at $\mathrm{m} / z \quad 192(100 \%)$ in addition to mass fragment peaks at $m / z 177$ (65\%), 164 $(30 \%)$ and $149(60 \%)$. The ${ }^{1} \mathrm{H}-\mathrm{NMR}$ spectrum revealed signals of two olefinic protons with cis-coupling at $\delta$ 6.27 and 7.60 (each $1 \mathrm{H}, \mathrm{d}, J=9.5 \mathrm{~Hz}$ ) indicative of the $\mathrm{H}-3$ and $\mathrm{H}-4$ of the coumarin nucleus; ${ }^{37}$ two aromatic protons (para to each others) at $\delta$ 6.84 and 6.91 (each 1H, s, H-5, H-8) and a singlet for one methoxyl group at $\delta 3.95(3 \mathrm{H}) .{ }^{13} \mathrm{C}-\mathrm{NMR}$ spectrum exhibited signals for 10 carbon atoms including a signal for $\delta$-lactone function at $\delta$ 161.47. These spectral data showed good agreement with those reported for scopoletin. ${ }^{37 \& 38}$ Thus, compound $\mathbf{1}$ was established as scopoletin.

Compound 2 possessed the molecular formula $\mathrm{C}_{16} \mathrm{H}_{14} \mathrm{O}_{4}$ by ESIMS that showed $[\mathrm{M}+\mathrm{H}]^{+}$at $\mathrm{m} / z$ 271 , together with mass fragment peaks at $m / z \quad 151(15 \%)$ and 147 (23\%). The UV spectrum exhibited two absorption bands at $\lambda_{\max } 240$ and $373 \mathrm{~nm}$ for chalcones. ${ }^{39}$ This was supported by the ${ }^{1} \mathrm{H}-\mathrm{NMR}$ spectrum which showed signals for two transolefinic protons at $\delta 7.46$ and 7.85 (each $1 \mathrm{H}, \mathrm{d}, J=15.5 \mathrm{~Hz}, \mathrm{H}-\alpha$ and $\beta$ ); 
two sets of ortho-coupled aromatic protons of B-ring at $\delta 6.89(2 \mathrm{H}, \mathrm{d}, J=$ $8.8 \mathrm{~Hz}, \mathrm{H}-3 / 5)$ and $7.58(2 \mathrm{H}, \mathrm{d}, J=$ $8.8 \mathrm{~Hz}, \mathrm{H}-2 / 6)$; three aromatic protons of A-ring at $\delta 6.48(1 \mathrm{H}, \mathrm{dd}$, $\left.J=9.2,1.6 \mathrm{~Hz}, \mathrm{H}-5^{\prime}\right), 6.96(1 \mathrm{H}, \mathrm{br} \mathrm{s}$, $\left.\mathrm{H}-3^{\prime}\right)$ and $7.83(1 \mathrm{H}, \mathrm{d}, J=9.2 \mathrm{~Hz}, \mathrm{H}-$ $6^{\prime}$ ) in addition to a singlet at $\delta 3.86$ $(3 \mathrm{H})$ for one methoxyl group. ${ }^{40}$ The dominent mass fragment peaks at $\mathrm{m} / \mathrm{z}$ 147 and 151 indicated the presence of one hydroxyl group in the B-ring in addition to one hydroxyl and one methoxyl group in the A-ring. ${ }^{41}$ The placement of the methoxyl group at position 4' was supported by the HMBC spectrum that displayed a correlation between the methoxyl protons $(\delta 3.86)$ and $C-4^{\prime}(\delta 166.4){ }^{41}$ Therefore, compound $\mathbf{2}$ was concluded to have the structure $4,2^{\prime}$ dihydroxy-4'-methoxychalcone (isoliquiritigenin 4'-methyl ether).

ESIMS of compound $\mathbf{5}$ showed $[\mathrm{M}+\mathrm{H}]^{+}$at $m / z 301$ for $\mathrm{C}_{16} \mathrm{H}_{12} \mathrm{O}_{6}$. It had a UV spectrum typical of an aurone having ortho-dihydroxyl groups on the B-ring and lacking a free hydroxyl group at C-4 since the complex formed with $\mathrm{AlCl}_{3}$ was acid labile. ${ }^{39}$ The ${ }^{1} \mathrm{H}-\mathrm{NMR}$ spectrum exhibited the pattern of aurones of the aureusidin type ${ }^{40}$ by the appearance of one singlet at $\delta 6.46(1 \mathrm{H}, \mathrm{s}, \mathrm{H}-10)$; three protons with the shifts and splitting of a catechol B-ring at $\delta 6.80$ $\left(1 \mathrm{H}, \mathrm{d}, J=8.2 \mathrm{~Hz}, \mathrm{H}-5^{\prime}\right), 7.16(1 \mathrm{H}$, $\left.\mathrm{dd}, J=8.2,1.9 \mathrm{~Hz}, \mathrm{H}-6^{\prime}\right)$ and 7.38 (1H, d, $\left.J=1.9 \mathrm{~Hz}, \mathrm{H}-2^{\prime}\right)$; two doublets at $\delta 6.14$ and 6.29 (each $1 \mathrm{H}, J=1.6$ $\mathrm{Hz}$ ) characteristic for meta-protons on
A-ring and a singlet at $\delta 3.82(3 \mathrm{H})$ for a methoxyl group. The ${ }^{13} \mathrm{C}-\mathrm{NMR}$ spectrum revealed the presence of 16 carbon atoms were attributed to aureusidin derivative. ${ }^{42}$ The methoxyl group was located at the 4-position according to long-range HMBC correlation between the methoxyl protons at $\delta 3.82$ and C-4 at $\delta 159.28$. On the basis of these data, compound 5 was shown to be aureusidin 4methyl ether (rengasin).

The EI mass spectrum of compound 8 showed $[\mathrm{M}]^{+}$at $\mathrm{m} / \mathrm{z} 194$ $(100 \%)$ corresponding to $\mathrm{C}_{10} \mathrm{H}_{10} \mathrm{O}_{4}$, in addition to mass fragment peaks at $\mathrm{m} / \mathrm{z} 179$ (42\%), 151 (10\%), 149 (9\%), $133(31 \%)$ and $77(10 \%)$. The ${ }^{1} \mathrm{H}-$ NMR spectrum showed signals of trans-3,4-disubstituted cinnamic acid: two olefinic protons with transcoupling at $\delta 6.23$ and 7.43 (each $1 \mathrm{H}$, $\mathrm{d}, J=16.0 \mathrm{~Hz}$ ); three aromatic protons at $\delta 6.93(1 \mathrm{H}, \mathrm{d}, J=8.2 \mathrm{~Hz}), 7.06$ $(1 \mathrm{H}, \mathrm{d}, J=1.9 \mathrm{~Hz})$ and $7.08(1 \mathrm{H}, \mathrm{dd}$, $J=8.2,1.9 \mathrm{~Hz}$ ) in addition to a signal of one methoxyl group at $\delta 3.79(3 \mathrm{H}$, $\mathrm{s})$. By comparison of these data with those published, ${ }^{43 \& 44}$ compound $\mathbf{8}$ was characterized as trans-4-hydroxy3-methoxycinnamic acid (transferulic acid).

Compounds 3, 4, 6, 7, 9, 10 and 12 displayed characteristic UV absorption maxima for flavone skeleton. ${ }^{39}$ The ${ }^{1} \mathrm{H}$-NMR spectral data for these compounds also confirmed the presence of flavone nucleus in these molecules. ${ }^{40}$

The ESI mass spectrum of compound 3 showed $[\mathrm{M}+\mathrm{H}]^{+}$at $\mathrm{m} / \mathrm{z}$ 
315 was consistent with the molecular formula $\mathrm{C}_{17} \mathrm{H}_{14} \mathrm{O}_{6}$. It had $\mathrm{UV}$ spectrum of a flavone having free hydroxyl groups at C-4' and C-7 and lacking a free hydroxyl group at $\mathrm{C}$ 5. ${ }^{39}$ The ${ }^{1} \mathrm{H}-\mathrm{NMR}$ spectrum exhibited the pattern of luteolin derivative ${ }^{40}$ by the appearance of a singlet aromatic proton at $\delta 6.55(1 \mathrm{H}, \mathrm{H}-3)$; two metacoupled protons in the A-ring at $\delta$ 6.41, 6.54 (each 1H, d, $J=2.2 \mathrm{~Hz}, \mathrm{H}-$ 6 and $\mathrm{H}-8$ ); three aromatic protons in the $3^{\prime}, 4^{\prime}$-disubstituted B-ring at $\delta 6.92$ $\left(1 \mathrm{H}, \mathrm{d}, J=8.2 \mathrm{~Hz}, \mathrm{H}-5^{\prime}\right), 7.45(1 \mathrm{H}, \mathrm{d}$, $\left.J=2.2 \mathrm{~Hz}, \mathrm{H}-2^{\prime}\right)$ and $7.47(1 \mathrm{H}, \mathrm{dd}, J=$ 8.2, $\left.2.2 \mathrm{~Hz}, \mathrm{H}-6^{\prime}\right)$ in addition to two singlets of two methoxyl groups at $\delta$ 3.88 and 3.95 (each $3 \mathrm{H}$ ). Considering the UV results, the two methoxyl groups could be placed at C-5 and C$3^{\prime}$. These placements are supported by the HMBC spectrum, the protons of the methoxyl groups at $\delta 3.88$ and 3.95 showed correlations with the carbons at position $5(\delta 161.5)$ and $3^{\prime}$ ( $\delta$ 148.6), respectively. By comparison of these spectral data with those in literature, ${ }^{45}$ compound $\mathbf{3}$ was deduced as luteolin 5,3'-dimethyl ether.

Compound 4 deduced the molecular formula $\mathrm{C}_{17} \mathrm{H}_{14} \mathrm{O}_{6}$ by ESIMS analysis $\left([\mathrm{M}+\mathrm{H}]^{+}\right.$at $\left.m / z 315\right)$. The UV spectral data suggested its structure to be a flavone having free hydroxyl groups at the 5- and 4'positions and lacking a free hydroxyl group at the 7-position. ${ }^{39}$ Its ${ }^{1} \mathrm{H}$-NMR spectral data were similar to those of compound 3. According to the UV spectral data, the methoxyl groups could be placed at positions 7 and 3'. On the basis of these spectral data and by comparison with the reported data, ${ }^{46}$ compound 4 was concluded to be luteolin 7,3'-dimethyl ether (velutin). ${ }^{46}$

The ESI mass spectra of compounds 6 and 7 showed $[\mathrm{M}+\mathrm{H}]^{+}$ at $m / z 271$ and 287 corresponding to the molecular formulae $\mathrm{C}_{15} \mathrm{H}_{10} \mathrm{O}_{5}$ and $\mathrm{C}_{15} \mathrm{H}_{10} \mathrm{O}_{6}$, respectively. They were characterized as apigenin and luteolin by comparison of their spectral data with published data. ${ }^{39,40,42 \& 47}$

ESIMS of compound 9 showed $[\mathrm{M}+\mathrm{H}]^{+}$at $\mathrm{m} / z 449$ corresponding to the molecular formula $\mathrm{C}_{21} \mathrm{H}_{20} \mathrm{O}_{11}$ in addition to a prominent fragment peak at $m / z 287$ [M-162 (hexose unit) $+\mathrm{H}]^{+}$indicating its monoside nature. The UV spectral data suggested its structure to be 5,7dihydroxyflavone derivative. ${ }^{39}$ In the ${ }^{1} \mathrm{H}$ - and ${ }^{13} \mathrm{C}-\mathrm{NMR}$ spectra, there were signals for luteolin nucleus ${ }^{40 \& 47}$ and a $\beta$-glucose moiety. ${ }^{48}$ In the HMBC spectrum the correlation from the glucose anomeric proton $\left(\mathrm{H}-\mathrm{1}^{\prime \prime}, \delta\right.$ $4.89)$ to $\mathrm{C}-4^{\prime}(\delta$ 148.58) confirmed the $O$-glycosidation at $\mathrm{C}-4^{\prime}$. Acid hydrolysis $^{31}$ of compound 9 yielded luteolin and glucose (co-TLC with authentic samples). On the basis of these results and by comparing the spectral data with literature, ${ }^{49}$ compound 9 could be identified as luteolin 4'-O- $\beta$-D-glucopyranoside.

Compound $\mathbf{1 0}$ had the same ESI mass spectrum of compound 9. Its UV spectra showed absorption pattern of 5,3',4'-trihydroxy flavone 
derivative. ${ }^{39}$ The ${ }^{1} \mathrm{H}$ - and ${ }^{13} \mathrm{C}-\mathrm{NMR}$ spectra were similar to those of compound 9. The HMBC spectrum showed correlation between the anomeric proton signal ( $\left.\delta 5.08, \mathrm{H}-1^{\prime \prime}\right)$ to $\mathrm{C}-7$ ( $\delta$ 162.94) confirmed the $O$ glycosidation at C-7. Acid hydrolysis $^{31}$ of compound $\mathbf{1 0}$ gave luteolin and glucose (co-TLC with authentic samples). From these results and comparison with literature data, ${ }^{39,40 \& 47}$ the structure of compound $\mathbf{1 0}$ was assigned as luteolin 7-O- $\beta$-D-glucopyranoside.

ESIMS of compound $\mathbf{1 2}$ showed $[\mathrm{M}+\mathrm{H}]^{+}$at $m / z 579$ for $\mathrm{C}_{27} \mathrm{H}_{30} \mathrm{O}_{14}$ in addition to a significant fragment peak at $m / z \quad 271$ [M-146 (methyl pentose unit)-162 (hexose unit) $+\mathrm{H}]^{+}$ indicating its bioside nature. UV spectral data of $\mathbf{1 2}$ suggested its structure to be 5,4'-dihydroxyflavone derivative. ${ }^{39}$ The ${ }^{1} \mathrm{H}$ - and ${ }^{13} \mathrm{C}-\mathrm{NMR}$ spectra displayed signals owing to apigenin, ${ }^{40 \& 47}$ one $\beta$-D-glucopyranosyl and one $\alpha$-L-rhamnopyranosyl ${ }^{48}$ units. The significant deshielding of the ${ }^{13} \mathrm{C}$ signal associated with $\mathrm{C}-2$ " of the glucose unit indicated further glucosylation at C-2". In the HMBC spectrum, the $1^{\prime \prime \prime} \rightarrow 2^{\prime \prime}$ link between the two sugars was confirmed by a ${ }^{3} \mathrm{~J}$ correlation from the anomeric proton $\delta 5.12$ ) of the rhamnose unit to 2 " ( $\delta$ $77.07)$ of the glucose unit. Also, the $O$-diglycosidic linkage at the 7position was supported from the correlation between the anomeric proton $(\delta 5.23)$ of the glucose unit and C-7 ( $\delta$ 162.59). Acid hydrolysis ${ }^{31}$ of compound $\mathbf{1 2}$ liberated apigenin, glucose and rhamnose (co-TLC with authentic samples). From these results and by comparison with the reported data, ${ }^{39,40 \& 47}$ compound $\mathbf{1 2}$ was identified as apigenin 7-O neohisperidoside.

The UV spectral data of compounds 11 and 14 suggested their structures to be flavonols with a substituted 3-hydroxyl group and free hydroxyl groups at positions 5, 7, 3' and $4^{\prime} .{ }^{39}$ By comparison of their ${ }^{1} \mathrm{H}$ NMR and ${ }^{13} \mathrm{C}-\mathrm{NMR}$ spectral data with those reported in literature, ${ }^{40 \& 47}$ compounds 11 and 14 were identified as quercetin 3-O- $\beta$-D-glucopyranoside and quercetin 3-Orutinoside (rutin), respectively.

The UV spectra of compounds $\mathbf{1 3}$ 15 and 16 exhibited characteristic absorption bands of flavonols with a substituted 3-hydroxyl group and free hydroxyl groups at the 5-, 7- and 4'positions. ${ }^{39}$ The ${ }^{1} \mathrm{H}-\mathrm{NMR}$ spectral data for these compounds supported their structures as kaempferol derivatives. ${ }^{40}$

Compound 13 showed $[\mathrm{M}+\mathrm{H}]^{+}$at $\mathrm{m} / \mathrm{z} 595$ in its ESI mass spectrum of corresponding to the molecular formula $\mathrm{C}_{27} \mathrm{H}_{30} \mathrm{O}_{15}$. Also, the spectrum revealed two prominent fragment peaks at $\mathrm{m} / \mathrm{z} 449$ [M-146 (methyl pentose unit) $+\mathrm{H}]^{+}$and 287 [M-146-162 (hexose unit) $+\mathrm{H}]^{+}$ indicating compound $\mathbf{1 3}$ as kaempferol 3-O-bioside. Its ${ }^{1} \mathrm{H}-\mathrm{NMR}$ spectrum displayed signals due to kaempferol skeleton ${ }^{40}$ together with two anomeric protons $[\delta 5.28(1 \mathrm{H}, \mathrm{d}$, $J=7.6 \mathrm{~Hz}$, Glc H-1) and 4.37 (1H, s, 
Rha H-1)]. ${ }^{48}$ Acid hydrolysis ${ }^{31}$ of compound $\mathbf{1 3}$ gave kaempferol, glucose and rhamnose (co-TLC with authentic samples). Thus, compound 13 was identified as kaempferol 3-Orutinoside.

The molecular formula of compound $\mathbf{1 5}$ was established as $\mathrm{C}_{32} \mathrm{H}_{38} \mathrm{O}_{19}$ by ESI mass analysis that showed $[\mathrm{M}+\mathrm{H}]^{+}$at $m / z$ 727. The three significant fragment peaks at $\mathrm{m} / \mathrm{z} 595$ $[\mathrm{M}-132 \text { (pentose unit) }+\mathrm{H}]^{+}, \quad 449$ [M-132-146 (methyl pentose unit) $+\mathrm{H}]^{+}$and 287 [M-132-146-162 (hexose unit) $+\mathrm{H}]^{+}$indicated compound 15 as kaempferol 3-Otriglycoside. The ${ }^{1} \mathrm{H}-\mathrm{NMR}$ spectrum exhibited signals of kaempferol ${ }^{40}$ in addition to three anomeric signals $[\delta$ $5.57(1 \mathrm{H}, \mathrm{d}, J=7.3 \mathrm{~Hz}$, Glc H-1), $4.59(1 \mathrm{H}, \mathrm{d}, J=7.3 \mathrm{~Hz}, \mathrm{Xyl} \mathrm{H}-1)$ and $4.34\left(1 \mathrm{H}, \mathrm{s}\right.$, Rha H-1)]. ${ }^{48}$ The high field position of $\mathrm{H}-1$ of $\beta$-xylose $(\delta$ 4.59) indicated that it was a terminal sugar. Because the $\mathrm{H}-1$ signal of the C-3 linked glucose was shifted downfield $(\Delta \delta=0.29 \mathrm{ppm})$ compared to that of rutinosyl unit in compound 13 it is suggested that xylose is attached to $\mathrm{C}-2{ }^{\prime \prime} .{ }^{50}$ The ${ }^{13} \mathrm{C}-\mathrm{NMR}$ spectrum displayed thirteen carbon signals were attributed to kaempferol nucleus ${ }^{42 \& 47}$ and seventeen carbon signals in the region of sugars including three anomeric signals at $\delta$ 104.49, 100.54 and 98.33 (see Table 1). The ${ }^{13} \mathrm{C}$-NMR shifts of the three sugars are consistent with those corresponding to one $\beta$-D-glucopyranosyl, one $\beta$-D-xylopyranosyl and one $\alpha$-L-rhamnopyranosyl moiety. ${ }^{48}$ The deshielding of the ${ }^{13} \mathrm{C}$ signals associated with $\mathrm{C}-2$ " $(\delta 81.66)$ and $C-6$ " $(\delta 66.23)$ of the glucose unit indicated the glycosylation at C-2" and $\mathrm{C}-6$ ". The identity of the three sugars and their sequence were determined by the exhaustive analysis of NMR spectral data (HMQC and HMBC). In the HMBC spectrum the anomeric proton signal at $\delta 5.57$ (Glc $\mathrm{H}-1)$ showed correlation with the carbon signal at $\delta 132.87$ (C-3) indicating the $O$-triglycosidic linkage at the 3-position of kaempferol. Also, the anomeric proton signal at $\delta 4.59$ (Xyl H-1) showed correlation with the carbon signal at $\delta 81.66\left(\mathrm{C}-2^{\prime \prime}\right)$ confirmed the interglycosidic linkage xylose $(1 \rightarrow 2)$ glucose. Furthermore, the correlation between the anomeric proton signal at $\delta 4.34$ (Rha H-1) and the carbon signal at $\delta 66.23\left(\mathrm{C}-6^{\prime \prime}\right)$ confirmed the interglycosidic linkage rhamnose $(1 \rightarrow 6)$ glucose. To confirm the nature and the absolute stereochemistry of the sugar moieties, an aliquot of $\mathbf{1 5}$ was hydrolysed and reacted with (-)-2-butanol. ${ }^{32} \mathrm{GC}$ analysis revealed the presence of Dglucose and D-xylose by comparison of their retention time data with those of the relevant standards. From the above evidences and by comparison with literature data, ${ }^{31850}$ compound 15 was established as kaempferol 3$O$-[2- $O$-D-xylopyranosyl-6- $O-\alpha$-Lrhamnopyranosyl]- $\beta$-D-glucopyrano$\operatorname{side}^{31}$ or kaempferol 3-[2 $2^{\mathrm{G}}$-xylosylrutinoside ${ }^{50}$ which is isolated here for the first time from family Cyperacea. It was previously reported 
from the leaves of Hosta ventricosa (Salisb) Stearn (Liliaceae) ${ }^{50}$ and seed cake of Camellia sinensis O. Kuntez (Theaceae). ${ }^{31}$

The ESI mass spectrum of compound 16 showed $[\mathrm{M}+\mathrm{H}]^{+}$at $\mathrm{m} / \mathrm{z}$ 757 corresponding to the molecular formula $\mathrm{C}_{33} \mathrm{H}_{40} \mathrm{O}_{20}$. Also, three prominent fragment peaks appeared at $595[\mathrm{M}-162 \text { (hexose unit) }+\mathrm{H}]^{+}, 449$ [M-162-146 (methyl pentose unit) $+\mathrm{H}]^{+}$and 287 [M-162-146-162 (hexose unit) $+\mathrm{H}]^{+} \quad$ indicating compound 16 as kaempferol 3-Otriglycoside. Its ${ }^{1} \mathrm{H}-\mathrm{NMR}$ spectrum exhibited signals owing to kaempferol $^{40}$ and three sugar units that were indicated by the appearance of three anomeric signals at $\delta 5.50$ $(1 \mathrm{H}, \mathrm{d}, J=7.0 \mathrm{~Hz}, \mathrm{Glc} \mathrm{H}-1), 4.58$ $(1 \mathrm{H}, \mathrm{d}, J=7.6 \mathrm{~Hz}, \mathrm{Glc} \mathrm{H}-1)$ and 4.29 $\left(1 \mathrm{H}, \mathrm{s}\right.$, Rha H-1). ${ }^{48}$ The strongest deshielded sugar doublet at $\delta 5.50$ apparently corresponding to the anomeric proton of glucose directly attached to C-3 position of the aglycone. ${ }^{50}$ The ${ }^{13} \mathrm{C}$-NMR spectrum displayed 30 carbon signals (see Table 1) were attributed to kaempferol, ${ }^{42 \& 47}$ two $\beta$-glucopyranosyl units and one $\alpha$-rhamnopyranosyl unit that were indicated by the appearance of three anomeric signals at $\delta 98.33,103.74$ and 100.42, respectively. ${ }^{48}$ The interglycosidic linkages at C-2" and C-6" were identified by the downfield shifts of $\mathrm{C}-2$ " ( $\delta$ 81.93) and C-6" ( $\delta$ 66.11). The HMQC and HMBC spectra identified the three sugars and their sequence. The HMBC spectrum showed a cross peak between the anomeric proton signal at $\delta 4.58\left(2^{\prime \prime}-\right.$ Glc $\mathrm{H}-1)$ and the carbon signal at $\delta$ 81.93 (3-Glc C-2"), this confirmed the interglycosidic linkage glucose $(1 \rightarrow 2)$ glucose. Also, the anomeric proton signal at $\delta 4.29$ (6"-Rha H-1) showed a cross peak with the carbon signal at $\delta 66.11$ (3-Glc C-6"), this supported the interglycosidic linkage rhamnose $(1 \rightarrow 6)$ glucose. Acid hydrolysis ${ }^{31}$ of compound $\mathbf{1 6}$ yielded kaempferol, glucose and rhamnose (co-TLC). The aforementioned data showed good agreement with those reported for kaempferol 3-O-[2-O-Dglucopyrano-syl-6- $O$ - $\alpha$-L-rhamnopyranosyl]- $\beta$-D-glucopyranoside or kaempferol 3-[2 ${ }^{\mathrm{G}}$-glucosylrutinoside ${ }^{50}$ which is isolated for the first time from the family Cyperaceae. It was previously reported from the leaves of Hosta ventricosa (Salisb) Stearn (Liliaceae). ${ }^{50}$

In conclusion, luteolin (7), luteolin 7-O- $\beta$-D-glucopyranoside (10) and quercetin 3-O-rutinoside (14) were previously reported from the leaves of the studied plant ${ }^{6}$ while the others are isolated for the first time from $C$. alpoecuroides Rottb. To the best of our knowledge, compounds $\mathbf{2}$ and $\mathbf{5}$ are first report from the genus Cyperus while compounds $\mathbf{1 5}$ and $\mathbf{1 6}$ are first report from the family Cyperaceae.

Flavonoids have the property of inhibiting autoxidation reactions and scavenging of free radicals but the relation between their structure and activity remains unclear. ${ }^{51}$ There are 
three functional groups that have been attributed to increase the antiradical activities among the flavonoids: the presence of an ortho-dihydroxylation of the B-ring of the flavonoid molecule; the $\mathrm{C} 2-\mathrm{C} 3$ double bond in concert with 4-oxo functionally of the C-ring; and the additional presence of both a 3- and a 5-hydroxyl moiety of the $\mathrm{C}$ and $\mathrm{A}$ rings, respectively. ${ }^{51 \& 52}$ Figure 2 shows the results obtained for antioxidant activity of fourteen flavonoids isolated from $C$. alopecuroides Rottb. Flavones and flavonols with a substituted hydroxyl group at the $\mathrm{C}-3$ position, which have only a C-4' hydroxyl group in the Bring showed low antioxidant activity falls within the range of $1.0-21.1 \%$. A strong antioxidant activity was shown by compounds with an orthodihydroxy system in the B-ring: quercetin 3-O- $\beta$-D-rutinoside (79.2\%), luteolin (75.0\%), luteolin 7$O$ - $\beta$-D-glucopyranoside $(71.9 \%)$ and quercetin $3-O-\beta$-D-glucopyranoside (70.7\%), aureusidin 4-methyl ether $(46.5 \%)$. It appears that the reason for their high reactivity is the strong effect of the C-3' hydroxyl group on the reactivity of the hydroxyl at C-4'. This is in agreement with conclusions that the ortho-dihydroxy system in the B-ring of the flavonoids is highly effective against free radicals. ${ }^{34 \& 51}$ The synergistic effects of flavonoid mixtures may be responsible for high antioxidant activity of the ethyl acetate, $n$-butanol and aqueous extracts $^{53}$ as shown in Figure 3.
The cytotoxicity of nine phenolic compounds isolated from $C$. alopecuroides Rottb. was assessed in vitro with a mouse lymphoma cell line using the microculture tetrazolium (MTT) assay. ${ }^{54}$ The compounds were tested for their cytotoxic activities at a range of concentrations of $(3-10 \mu \mathrm{g} / \mathrm{ml})$. All compounds were found to be active against the cell line chosen (Table 2). Luteolin 5,3'-dimethyl ether and luteolin 7,3'-dimethyl ether are the most active compounds in this study with $\mathrm{ED}_{50}$ of 2.7 and $3.2 \mu \mathrm{g} / \mathrm{ml}$, respectively.

Twelve flavonoids were evaluated for $\alpha$-amylase inhibitory activity. As shown in Figure 4, luteolin (99.6\%), aureusidin 4-methyl ether (90.5\%), luteolin 5,3'-dimethyl ether (85.0\%) and luteolin $4^{\prime}-O-\beta$-D-glucopyranoside $(83.8 \%)$ showed strong inhibitory activity of $\alpha$-amylase. The $\alpha$-amylase inhibitory activity of luteolin $\left(\mathrm{IC}_{50} \quad 50-125 \mu \mathrm{g} / \mathrm{ml}\right)$ was similar to acarbose ( $\mathrm{IC}_{50} \quad 50-120$ $\mu \mathrm{g} / \mathrm{ml})$. Apparently, the $\alpha$-amylase inhibitory activity increased with the presence of hydroxyl groups at $3^{\prime}$ and $4^{\prime}$-position of the B-ring. ${ }^{55}$

In conclusion, the present study revealed that the titled plant contains a large number of phenolics with strong antioxidant, cytotoxic activities and an inhibitory activity of $\alpha$ amylase. Thus, the plant could be considered of potential impact on human health. 
Table 2: Cytotoxic activity of some phenolic compounds isolated from the inflorescences of $C$. alopecuroides Rottb.

\begin{tabular}{|c|c|}
\hline Compound & $\mathrm{ED}_{50}(\mu \mathrm{g} / \mathrm{ml})$ \\
\hline Luteolin 5,3'-dimethyl ether (3) & 2.7 \\
\hline Luteolin 7,3'-dimethyl ether (4) & 3.2 \\
\hline Aureusidin 4-methyl ether (5) & 7.2 \\
\hline Trans-ferulic acid (8) & $>10.0$ \\
\hline 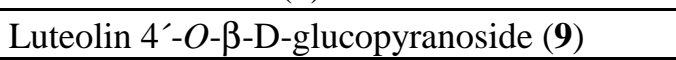 & $>10.0$ \\
\hline Luteolin 7-O- $\beta$-D-glucopyranoside (10) & $>3.0$ \\
\hline Kaempferol 3-O- $\beta$-D-rutinoside (13) & 8.8 \\
\hline Kaempferol 3-[2 $2^{G}$-xylosylrutinoside] (15) & $>10.0$ \\
\hline Kaempferol 3-[ $2^{\mathrm{G}}$-glucosylrutinoside] (16) & $>10.0$ \\
\hline
\end{tabular}

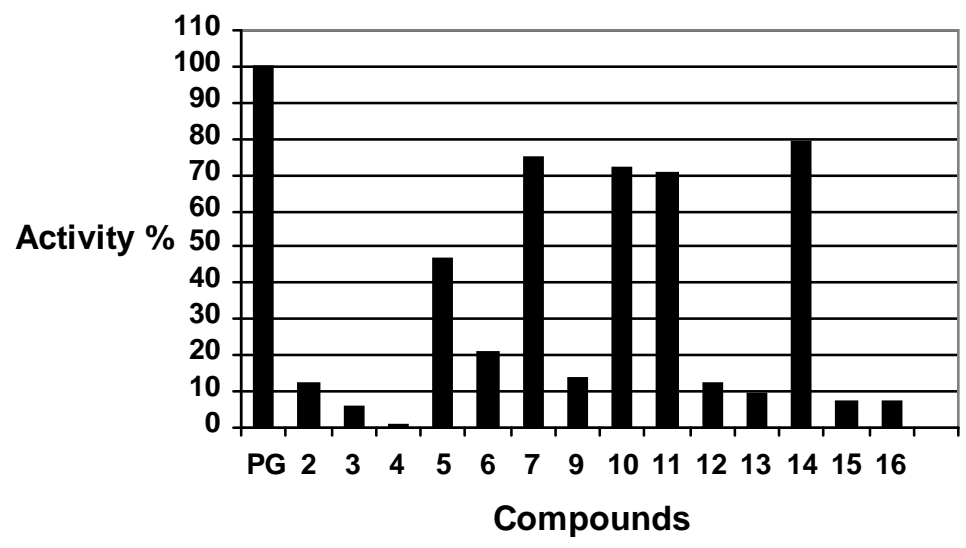

Fig. 2: Antioxidant activity of some flavonoids isolated from the inflorescences of $C$. alopecuroides Rottb. 


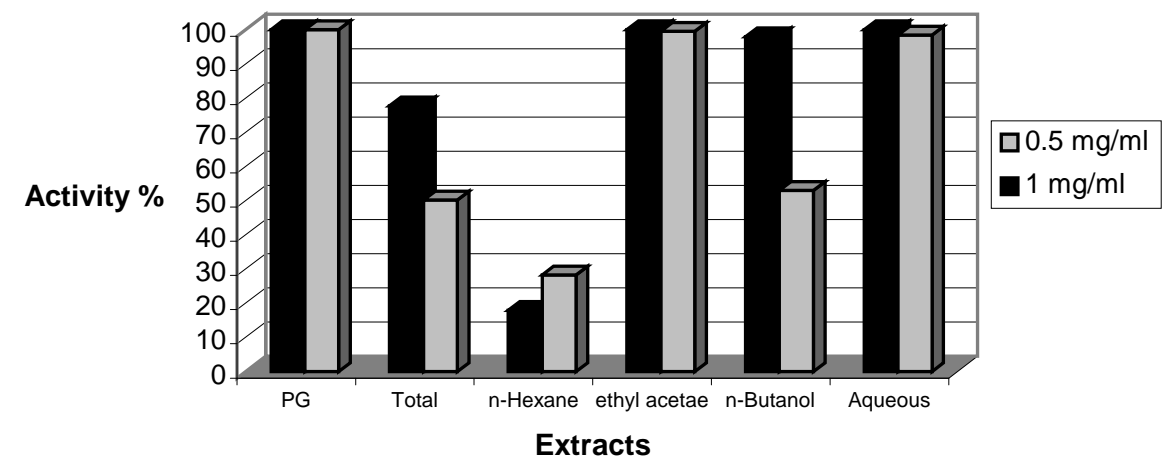

Fig. 3: Antioxidant activity of the different extracts from the inflorescences of $C$. alopecuroides Rottb.

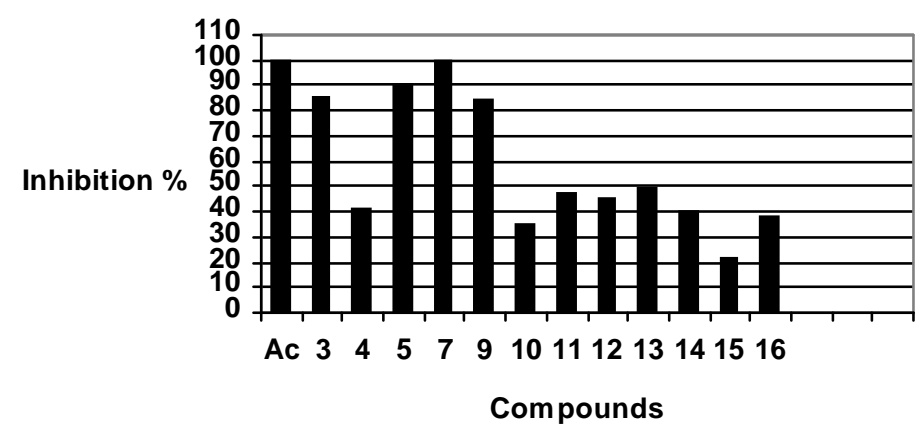

Fig. 4: $\alpha$-Amylase inhibitory activity of some flavonoids isolated from the inflorescences of $C$. alopecuroides Rottb. 


\section{Acknowledgement}

We are indebted to Prof. Dr. W. E. G. Müller (Institute für Physiologische Chemie, Duesbergweg 6, D-55099 Mainz, Germany) for cytotoxicity testing.

\section{REFERENCES}

1- W. C. Evans, "Trease and Evans Pharmacognosy", $15^{\text {th }}$ Ed., W. B. Saunders, London, 2002, p. 38.

2- L. Boulos and M. N. El-Hadidi, "The Weed Flora of Egypt", The American University in Cairo Press, Cairo, 1984, p. 58.

3- R. D. Allan, R. J. Wells, R. L. Correll and J. K. Macleod, Phytochemistry, 17, 263 (1978).

4- M. Morimoto, Y. Fujii and K. Komai, ibid., 51, 605 (1999).

5- A. C. Alves, M. M. Moreira, M I. Paul and M. A. C. Costa, ibid. 31, 2825 (1992).

6- I. El-Habashy, R. M. A. Mansour, M. A. Zahran, M. N. El-Hadidi and N. A. M. Saleh, Biochemical Systematics and Ecology, 17, 191 (1989).

7- J. B. Harborne, C. A. Williams and K. L. Wilson, Phytochemistry, 21, 2491 (1982).

8- M. Abdel-Mogib, S. A. Basaif and S. T. Ezmirly, Pharmazie, 55, 693 (2000).

9- R. M. Seabra, A. M. S. Silva, P. B. Andrade and M. M. Moreira, Phytochemistry, 48, 1429 (1998).
10- A. S. Awaad and M. E. Zain, Egypt. J. Pharm. Sci., 40, 107 (1999).

11- A. Dini, E. Ramundo, P. Saturnino, A. Scimone, I. Stagno d'Alcontres, Boll. Soc. Ital. Biol. Sper., 68, 453 (1992).

12- H. M. Sayed, M. H. Mohamed, S. F. Farag and G. A. Mohamed, Bull. Fac. Pharm. Cairo Univ., 39, 195 (2001).

13- H. Hikino and K. Aota, Phytochemistry, $15, \quad 1265$ (1976).

14- B. Nyasse, R. G. Tih, B. L. Sondengam, M. T. Martin and B. Bodo, ibid., 27, 179 (1988).

15 - M. M. Sonwa, W. A. König, K. Kubeczka and O. Motl, ibid., 45, 1435 (1997).

16- B. M. Fraga, Natural Product Reports, 9, 217 (1992).

17- $\mathrm{F} . \mathrm{Xu}, \mathrm{T}$. Morikawa, $\mathrm{H}$ Matsuda, K. Ninomiya and M. Yoshikawa, J. Nat. Prod., 67, 569 (2004).

18- S. Jeong, T. Miyamoto, M. Inagaki, Y. Kim and R. Higuchi, ibid., 65, 673 (2000).

19- P. N. Singh and S. B. Singh, Phytochemistry, 19, 2056 (1980).

20- A. Kar and C. I. Okpaleke, Journal of African Medicinal Plants, 5, 189 (1982).

21- K. Koichiro and U. Kunikazu, Shokubutsu No Kagaku Chosetsu, 16, 32 (1981). Through C. A., 97 (15) 123942u (1982).

22- L. Boulos, "Medicinal Plants of North Africa", Reference 
Publications, Michigan, 1983, pp. 80-2.

23- B. Oliver-Bever, "Medicinal Plants in Tropical West Africa", Cambridge University Press, Cambridge, 1986, pp. 144- 217.

24- L. M. Perry and J. Metzger, "Medicinal Plants of East and Southeast Asia: Attributed Properties and Uses", MIT Press, London, 1980, pp. 12122.

25- A. Chevallier, "The Encyclopedia of Medicinal Plants", Dorling Kindersley Ltd., London, 1996, p. 197.

26- M. S. Hifnawy, Y. Y. ElHyatmy, S. A. Kenawy, A. K. Yossef and A. S. Awaad, Bull. Fac. Pharm. Cairo Univ., 37, 99 (1999).

27- M. S. Hifnawy, H. H. Ammar, S. K. Kenawy, M. E. Zaki, A. K. Yossef and A. S. Awaad, ibid., 37, 107 (1999).

28- M. I. Nassar, A. F. Abdel-Razik, E. A. M. El-Khrisy, A. M. Dawidar, A. Bystrom and T. J. Mabry, Phytochemistry, 60, 385 (2002).

29- H. M. A. El-Gohary, Bull. Fac. Pharm. Cairo Univ., 42, 157 (2004).

30- M. M. Sonwa and W. A. König, Phytochemistry, 56, 321 (2001).

31- T. Sekine, J. Arita, A. Yamaguchi, K. Saito, S. Okonogi, N. Morisaki, S. Jwasaki and I. Murakoshi, ibid., 30, 991 (1991).

32- M. Fouad, K. Al-Trabeen, M. Badran, V. Wray, R. A. Edrada,
P. Proksch and R. Ebel, Arkivoc, xiii, 17 (2004).

33- B. Steffan, W. Wätjen, G. Michels, P. Niering, V. Wray, R. Ebel, R. A. Edrada, R. Kahl and P. Proksch, J. Pharm. Pharmacol., 57, 233 (2005).

34- M. Joyeux. A. Lobstein, R. Anton and F. Mortier, Planta Med., 61, 126 (1995).

35- R. A. Edrada, P. Proksch, V. Wray, L. Witte, W. E. Müller and R. W. M. Van Soest, J. Nat. Prod., 59, 1056 (1996).

36- Molecular Probes website: www.probes.com.

37- S. Öksüz, A. Ulubelen, A. Barla and W. Voelter, Turk. J. Chem. 26, 457 (2002).

38- J. M. J. Vasconcelos, A. M. S. Silva and J. A. S. Cavaleiro, Phytochemistry, 49, 1421 (1998).

39- T. J. Mabry, K. R. Markham and M. B. Thomas, "The Systematic Identification of Flavonoids", Springer, New York (1970).

40- J. B. Harborne, "The Flavonoids: Advances in Research Since 1986", Chapman and Hall, London (1994)

41- V. Seidel, F. Bailleul and P. G Waterman, Phytochemistry, 55, 439 (2000).

42- P. K. Agrawal, "Carbon-13 NMR of Flavonoids", Elsevier Science, New York, Tokyo (1989).

43- H. Tominaga, Y. Kobayashi, T. Goto, K. Kasemura and M. Nomura, Yakugaku Zasshi, 125, 371 (2005). 
44- A. M. Dawidar, S. T. Ezmirly, M. Abdel-Mogib, Y. ElDessouki and R. F. Angawi, Pharmazie, 55, 848 (2000).

45- H. Achenbach, M. Stöcker and M. A. Constenla, Phytochemistry, 27, 1835 (1988).

46- M. Sakakibara, D. Jr. Difeo, N. Nakatani, B. Timmermann and T. J. Mabry, ibid., 15, 727 (1976).

47- J. B. Harborne and T. J. Mabry, "The Flavonoids: Advances in Research", Chapman and Hall, London (1982).

48- P. K. Agrawal, Phytochemistry, 31, 3307 (1992).

49- M. Yoshizaki, H. Fujino, M. Masuyama, M. Arisawa and N. Morita, ibid., 26, 2557 (1987).

50- J. Budzianowski, ibid., 29, 3643 (1990).
51- S. Burda and W. Oleszek, J. Agric. Food Chem., 49, 2774 (2001).

52- A. J. Jr. Dugas, J. CastanedaAcosta, G. C. Bonin, K. L. Price, N. H. Fischer and G.W. Winston, J. Nat. Prod., 63, 327 (2000).

53- J. B. Harborne and C. A. Williams, Phytochemistry, 55, 481 (2000).

54- M. H. Kreuter, A. Robitzki, S. Chang, R. Steffen, M. Michaelis, Z. Kljajic, M. Bachmann, H. C. Schröder and W. E. G. Müller, Comp. Biochem. Physiol., 101 C, 183 (1992).

55- T. Matsui, M. Kobayashi, S. Hayashida and K. Matsumoto, Biosci. Biotechnol. Biochem. 66, 689 (2002). 